Purdue University Purdue e-Pubs

ECE Technical Reports

Electrical and Computer Engineering

$11-1-1993$

\title{
On Efficient and Robust Methods Of Accurate Camera Calibration
}

Chanchal Chatterjee

Purdue University School of Electrical Engineering

Vwani P. Roychowdhury

Purdue University School of Electrical Engineering

Follow this and additional works at: http://docs.lib.purdue.edu/ecetr

Chatterjee, Chanchal and Roychowdhury, Vwani P., "On Efficient and Robust Methods Of Accurate Camera Calibration" (1993). ECE Technical Reports. Paper 252.

http://docs.lib.purdue.edu/ecetr/252

This document has been made available through Purdue e-Pubs, a service of the Purdue University Libraries. Please contact epubs@purdue.edu for additional information. 


\section{ON EFFICIENT AND ROBUST Methods of AcCurate Camera Calibration}

Chanchal Chatterjee

VWANI P. ROYCHOWDHURY

TR-EE 93-45

NOVEMBER 1993

SCHOOL OF EleCtrical ENGINEERING PURDUE UNIVERSITY WEST LAFAYETTE, INDIANA 47907-1285 


\title{
On Efficient and Robust Methods \\ Of Accurate Camera Calibration
}

\author{
Chanchal Chatterjee \\ and \\ Vwani P. Roychowdhury
}

School of Electrical Engineering

Purdue University

West Lafayette, IN 47907-1285 
Title

ON EFFICIENT AND ROBUST METHODS OF

ACCURATE CAMERA CALIBRATION

\begin{abstract}
Authors
Chanchal Chatterjee* and Vwani Roychowdhury

School of Electrical Engineering, Purdue University,

West Lafayette, Indiana 47907
\end{abstract}

\begin{abstract}
In this paper we have presented a novel algorithm for camera calibration which is a significant improvement in mathematical simplicity, accuracy and computational efficiency in the solution of all extrinsic (external camera geometric) and intrinsic (internal camera geometric and camera optics) parameters. The method involves a direct transformation from the threedimensional (3D) object world to the two-dimensional (2D) image or sensor plane in terms of "homogeneous vector forms" for the solution of 12 extrinsic and a number of intrinsic parameters for both coplanar and non-coplanar distributions of object points. Furthermore, we have demonstrated a strong robust property of the proposed algorithm by proving (with experimental corroboration) that if the camera is calibrated with image data not compensated for image center displacement and scale factor, the proposed algorithm yields parameters that cause no errors in the computation of both image and world coordinates. In addition, we have discussed a new method of parameter computation under a complete lens distortion effect (including both radial and tangential distortions) by the method of constrained least squares. Analytical proofs of convergence are also given. Moreover, we have provided a new complete algorithm for the solution of all calibration parameters. Finally, we have proposed a new Incremental Model for the correspondence of tolerances between the 3D object world and the 2D image plane with and without intrinsic parameter effects. Experimental results on a coplanar set of object points have been provided to support our models.
\end{abstract}

Chanchal Chatterjee is also Project Manager of Vision
Systems at Phoenix Software Development Co., Sterling Heights, MI. 


\subsection{Introduction}

Camera Calibration is an important task in the field of machine vision applications that require:

(1) conversion of real-world coordinates of object points into pixel coordinates of the corresponding points in an image,

(2) conversion of pixel coordinates of image points into realworld coordinates of the corresponding object features,

(3) calculation of various extrinsic (external camera geometric) and intrinsic (internal camera geometric or camera optics) parameters,

(4) conversion between real-world tolerances and pixel distances.

In most state of the art models and algorithms $[6,8-10,15,21-28]$ for camera calibration, we can find discussions on the first three tasks above. In this paper we shall establish new methods for fast, robust and accurate solutions to these tasks, and also present new models for efficient computation of the fourth.

In classical image processing applications such as part dimensional measurement $[19,20]$, robot calibration $[3,7,12,21]$, and automatic assembly of mechanical or electronics components $[2,11]$, camera calibration is usually performed at the learn or teach phases where the speed of the calibration method is not very critical. However, in many real-time applications such as object tracking and trajectory analysis, the speed of the camera calibration method is important for efficient run-time performance. It is generally agreed $[10,13,23,28]$ that any technique involving non-linear optimization $[9,21,26,27]$ that require computer-intensive full-scale search is too complex for these real-time applications. For these applications and others, we shall focus on novel algorithms for high speed performance with a high degree of accuracy.

\subsection{Brief Review of State of the Art:}

A well-known model for calibration of extrinsic camera parameters to obtain three-dimensional (3D) measurements from a stereo pair of cameras has been developed by Yakimovsky and 
Cunningham[28] for the JPI Robotics Research Laboratory. A direct transformation from the 3D world coordinates to the twodimensional (2D) image coordinates has been established in this approach resulting in linear equations whose solutions yield the unknown camera parameters. A direct advantage of this method is that a closed form solution is obtained for all extrinsic parameters and focal length. However, due to a narrow field of view and large object distances they used a linear lens model and ignored distortions caused by non-linearities in the lens systems. All intrinsic parameters except focal length are ignored. The basic camera geometry in this model has been used to build our analyses and algorithms. However, as discussed in Section 1.2, we have significantly improved several aspects of this basic model.

An alternative exhaustive approach to camera calibration has been presented by Tsai[13,23] which first transforms the 3D object coordinates to a 3D space and subsequently projects it to the 2D image or sensor plane. The advantages of this method are as follows: (a) by the two-step coordinate transformation Tsai has successfully reduced the computational complexity of the calibration algorithm, because most of the parameters are computed by a single step least squares approach, and (b) some intrinsic parameters such as image center displacement, scale factor and radial lens distortion are considered for higher accuracy. The disadvantages of the method are as follows: (a) the method considers only radial lens distortion and cannot be extended to any other types of distortion, and (b) the method tends to ignore constraints which the extrinsic and intrinsic parameters must obey. It has been argued in [25] that this "solution is not optimal because the information provided by the calibration points has not been fully utilized".

Grosky and Tamburino[10] have presented a method of camera calibration that uses a linear affine transformation as a map between the camera coordinates and the geometrically projected coordinates on the image plane of known reference points. The advantages of this method are as follows: (a) all parameters are solved within the specified constraints, and (b) many intrinsic 
parameters such as scaling, aspect ratio, displacement of image center and skew angle are considered. The disadvantages of the method are as follows: (a) lens distortion is solved geometrically by transforming the distorted image to a rectangular camera grid; lens distortion is, therefore, not considered as a camera parameter that is calibrated with the remaining intrinsic parameters and (b) as the computational complexity of the method is simplified, in some cases, simpler solutions can be obtained at the price of lower accuracy.

A method of 3D position sensing using a calibration scheme that relates depth to focus blur has been presented by Cardillo and Ahmed[6]. The main advantage of this method is the computational efficiency. The disadvantages of this method are as follows: (a) the method assumes sharp scene edges when measuring blur; noise and lighting intensity variations adversely affect the performance of this method, and (b) the method ignores effects of lens distortion in the measurement of blur ledges.

Wang and Tsai[24] have presented a method of camera calibration that demonstrates that vanishing lines include useful geometric hints about the camera extrinsic parameters and the focal length, from which these parameters can be solved easily and analytically. This technique uses multiple steps of efficient least squares method for parameter computation. No intrinsic parameter is solved by this method except for focal length.

A method of camera calibration with a complete lens distortion model is presented by Weng, et al[25]. This method proposes multiple steps of iterative linear and non-linear optimization schemes to solve all extrinsic and intrinsic camera parameters. The advantages of this method are as follows: (a) the method solves all calibration parameters, and (b) the parameters are solved within specified constraints. The disadvantages are as follows: (a) the method uses multiple iterative algorithms at the cost of computational efficiency, and (b) in its iterative procedure, the method decouples the lens distortion parameters from the remaining extrinsic and intrinsic parameters in order to solve one while the other is kept fixed; this assumes that the 
lens distortion parameters can be de-coupled from the :remaining parameters without giving false minima in parameter computation.

\subsection{Comparison with State of the Art:}

In this paper, we have developed new algorithms for camera calibration that has the following enhanced features:

\section{(1) Computational Efficiency and Mathematical Simplicity:}

As mentioned before, alternative efficient methods for camera calibration have been presented by many recent researchers $[10,13,23,28]$. Our new proposed algorithm (Section 2) improves the mathematical simplicity of the steps involved in the computation of calibration parameters, while enhancing the computational efficiency when compared to current methods. The enhanced simplicity is due to the direct transformation between the 3D object world and the 2D image plane.

In other words, while enhancing the mathematical simplicity of the proposed algorithm, we have attained the computational efficiency of the most efficient current technique $[13,23]$. Moreover, our approach leads to several desirable properties such as robustness (Section 3) and appropriate framework forc the solution of all extrinsic and intrinsic parameters (Sections 4 and 5).

\section{(2) Robustness of Calibration Algorithm:}

In most state of the art calibration methods, it is important to compute the intrinsic parameters such as image center displacement and scale factor $[10,13,18,23,24]$ for accurate calibration results. In this paper, we have analyzed the influence of both image center displacement and scale factor on the extrinsic parameters computed by the proposed algorithm, and also on the computation of image and world coordinates (refer Section 3). We have demonstrated the following results:

(i) Our analysis shows that both image center displacement and 
scale factor have no effect (in the coplanar and noncoplanar calibration cases) on the computation of both image and world coordinates, if the camera is calibrated with the uncompensated image data using our proposed algorithm. This result allows us to perform accurate camera calibration without extensive computation of intrinsic parameters such as image center displacement and scale factor.

\section{(ii) We further demonstrate the influence of these intrinsic parameters on the extrinsic parameters computed by our algorithm. This analysis allows us to adjust the extrinsic parameters based on these intrinsic parameter values, and reduces the task of extensive re-computation of the extrinsic parameters when the image center or scale factor changes.}

Some researchers $[13,23]$ have observed, through experimental studies, that image center displacement has minimal effect on camera calibration for the non-coplanar case. For example, in [23] the following observation is made: "To see the consequence of having a wrongly guessed image center when doing calibration, we intentionally altered the apparent image center by ten pixels. The results of 3D measurement still is about as accurate". We have confirmed this fact by analytical means and also extended it to the coplanar distribution of object points. Furthermore, we have extended this result to include the scale factor parameter. This appears to be the first known analvtical result of its kind in a calibration algorithm.

\section{(3) Complete Lens Distortion:}

In most camera calibration methods commonly used in applications, the effects of image distortion due to non-linear lens systems are ignored. However, many researchers $[12,15]$ have observed that ignoring lens distortion is unacceptable in doing 3D measurements. Many recent studies $[10,13,18,23]$ have considered new methods for correcting lens distortion. These methods, although very accurate, mostly consider radial distortions of lenses. When complete lens distortion is considered $[25,26]$, 
computationally intensive iterative linear and non-linear optimization schemes are used.

We have presented a new algorithm to solve the complete lens distortion problem with both radial and tangential distortions of lenses by extending the least squares solution of linear equations to a constrained least squares solution by the use of Lagrange multipliers[14]. We have also included analytical proofs and experimental results for the convergence of the proposed iterative algorithm. While other researchers have previously presented iterative algorithms, our analysis appears to be the onlv one that shows an analytical proof of convergence of this algorithm.

(4) Complete Algorithm for the Solution of All Parameters:

A direct outcome of the robustness and the lens distortion algorithms is a complete algorithm for the solution of all calibration parameters. Although state of the art procedures[25] exist for such solutions, we have discussed the robustness of our approach in comparison with these procedures. We have partitioned the parameter space into two subsets for the solution of all extrinsic and intrinsic parameters, and have argued that our new procedure is more robust and less sensitive to noise and starting values in iteration.

\section{(5) Incremental Model:}

In this paper we have also studied an aspect of camera calibration that commonly occur in machine vision applications involving measurements and tolerance computations such as defect measurement and robotic part placement. In such applications it may be sufficient to compute the correspondence between distances (or tolerances) in 3D to distances in pixels. For these situations, we have developed a special correspondence model called the Incremental Model. We shall present the Incremental Model both with and without the influence of intrinsic parameters (such as lens distortion) for coplanar and non-coplanar distributions of object points. 
We have established a direct transformation between the 3D object world and the 2D image or sensor plane in terms; of "homogereous vector forms". In most state of the art models, the homogeneous transformation is between the 3D object world and a 3D camera space, which is subsequently projected to the image or sensor plane. This is a two-step transformation. The proposed one-step homogeneous transformation yields a simple set of mathematical steps for the solution of all calibration parameters, and demonstrates a geometrical significance of the calibration parameters in the physical camera setup. Furthermore, we have carried the analysis for both coplanar and non-coplanar distributions of object points, and have provided exhaustive solutions for both.

In summary, our approach to camera calibration is a significant improvement in mathematical simplicity, accuracy, and computational efficiency in the solution of all extrinsic and intrinsic parameters. Furthermore, this method leads to a robust solution of calibration parameters, where the image center displacement and scale factor have no effect on the computation of image! or world coordinates. Also we have demonstrated analytically, the effects of image center displacement and scale factor on the extrinsic parameters. In addition, we have considered complete lens distortion, and added the new aspect of the Incremental Model. A new iterative constrained least squares approach to compute lens distortion parameters with an analytical proof of convergence is also studied. Finally, a new complete algorithm for the solution of all calibration parameters, as shown in. Table 1, is presented. 
TABLE 1. Camera Calibration Parameters Discussed in this Study.

\begin{tabular}{|c|c|c|}
\hline Params & Type & Description \\
\hline $\begin{array}{l}\hat{\mathrm{H}}_{x}, \hat{\mathrm{H}}_{y}, \hat{\mathrm{H}}_{\mathrm{z}} \\
\hat{\mathrm{V}}_{\mathrm{x}}, \hat{\mathrm{V}}_{\mathrm{y}}, \hat{\mathrm{V}}_{\mathrm{z}} \\
\hat{\mathrm{A}}_{x}, \hat{\mathrm{A}}_{y}, \hat{\mathrm{A}}_{\mathrm{z}} \\
\mathrm{C}_{\mathrm{h}}, \mathrm{C}_{\mathrm{v}}, \mathrm{C}_{\mathrm{a}}\end{array}$ & Extrinsic & $\begin{array}{l}\text { Horizontal camera vector. } \\
\text { Vertical camera vector. } \\
\text { Axial camera vector. } \\
\text { Position of camera center. } \\
\text { New efficient computational methods are } \\
\text { presented. }\end{array}$ \\
\hline $\mathbf{f}$ & Intrinsic & $\begin{array}{l}\text { Focal Length. } \\
\text { New efficient computational methods are } \\
\text { presented. Pertains to the physical } \\
\text { camera optics. }\end{array}$ \\
\hline $\begin{array}{l}i_{0}, j_{0} \\
s_{i}, s_{j}\end{array}$ & Intrinsic & $\begin{array}{l}\text { Image Center Displacement. } \\
\text { Scale Factor. } \\
\text { Effects on extrinsic parameters and } \\
\text { coordinate computation have been } \\
\text { demonstrated analytically. }\end{array}$ \\
\hline$l_{1}, p_{1}, p_{2}$ & Intrinsic & $\begin{array}{l}\text { Lens Distortion. } \\
\text { Complete lens distortion is considered. } \\
\text { Efficient constrained least squares } \\
\text { solution is proposed. Analytic } 1 \text { proof } \\
\text { of convergence provided. }\end{array}$ \\
\hline
\end{tabular}

\subsection{Outline of the Paper:}

In Section 2 we shall present the camera calibration model and represent the correspondence between the world and image coordinates in a "homogeneous vector form". A novel algorithm for extrinsic parameter (and focal length) computation will be presented for both coplanar and non-coplanar distributions of object points. In section 3 the influence of image center displacement and scale factor on the extrinsic parameters and, on coordinate computation will be discussed. The robustness of the proposed algorithm will also be analyzed. In Section 4, we shall 
propose a new algorithm for complete corrections of lens distortion effects for enhanced calibration accuracy. Analytical proof of convergence of this algorithm will be shown.

In Section 5 a complete algorithm for the solution of all calibration parameters will be presented. Robustness of the complete algorithm in comparison with state of the art methods will be discussed. Section 6 will contain an error analysis of the calibration parameters. The effects of measurement. errors of image ccordinates on the calibration parameters will be analyzed. Section 7 will discuss the new Incremental Model with and without the influence of intrinsic parameters for both coplanar and noncoplanar cases. Section 8 will contain experimental results on a coplanar set of object points. Section 9 will have concluding remarks.

\subsection{Camera Calibration Model}

This section of the paper deals with the geometrical aspects of the calibration problem. The geometry assumes that the camera and lens system behave like a pinhole camera system without any distortion. The effects of image distortions due to lens configuration have been added to this geometrical system for improved. accuracy in the next sections (Section 3 and 4 ). The geometry involves essentially three coordinate systems (refer Figure 1) :

(1) a 3D world coordinate system $(X, Y, Z)$ centered around a point $W$ and including a point $P(x, y, z)$ in $3 D$,

(2) a 2D image or sensor array system ( $I, J)$ centered at a point $S$ in the sensor plane, and describing the "horizontal" and "vertical" positions of image pixels,

(3) an intermediate 3D coordinate system ( $\mathrm{B}, \mathrm{V}, \mathrm{A})$ with origin at optical center $\mathrm{C}$ of the lens system, and describing the "horizontal", "vertical" and "axial" directions of the camera system. 
The world coordinate system is arbitrary and is often dictated by the application. However, it is assumed in our discussions that the coordinate systems are right-handed, and that the axes are mutually orthogonal. The intermediate coordinate system is often referred to as the camera coordinate system. The $\mathrm{H}-\mathrm{V}$ plane of the camera coordinate system is viewed as being parallel to the sensor array plane, with the $\mathrm{H}$ and $\mathrm{V}$ axes aligned respectively to the $I$ and $J$ axes of the sensor coordinate system. In a purely geometrical model, the third axis A of the camera coordinate system lies on the optical axis of the lens package. The sensor coordinate system $(I, J)$ is centered at a point $S$ on the sensor plane lying on the optical axis of the lens package, i.e. along camera axis $A$.

Establishing a relationship among these three coordinate systems is the central issue within the camera calibration problem. Let $(\hat{B}, \hat{V}, \hat{A})$ be unit vectors for the camera coordinate system $(B, V, A)$. A point $P$ in $3 D$ with world coordinates $(X, Y, z)$ is projected through the optical center $C$ onto the sensor array at a point $(i, j)$ in the sensor (image) coordinates (refer Figure 1 ). Let $f$ be the distance from optical center $C$ to the sensor plane. By similar triangles:

$$
\begin{aligned}
& \frac{(P-C) \cdot \hat{H}}{(P-C) \cdot \hat{A}}=\frac{i}{f} \\
& \frac{(P-C) \cdot \hat{V}}{(P-C) \cdot \hat{A}}=\frac{j}{f}
\end{aligned}
$$

Dividing equation ( 1 ) by equation (2), and assuming $C_{h}=C$. $\hat{B}$, $C_{v}=C \cdot \hat{v}$ and $C_{a}=C \cdot \hat{A}$, we create the following matrix equation: 


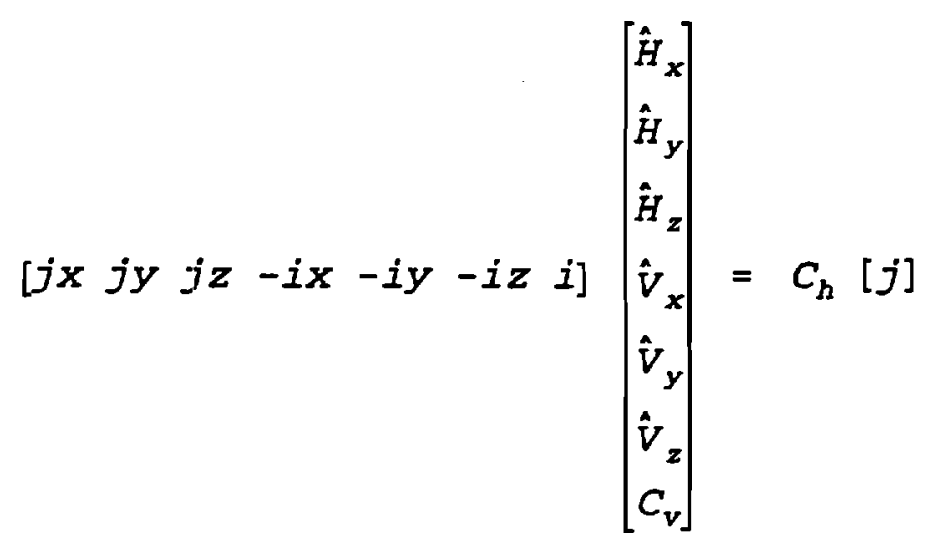

In equation (3) $(i, j)$ are measured pixel coordinates of a point $P$ in $3 \mathrm{D}$ with known coordinates $(x, y, z)$. The camera parameters $\hat{\mathrm{H}}_{\mathrm{x}}$, $\hat{\mathrm{H}}_{y}, \hat{\mathrm{H}}_{z}, \hat{\mathrm{V}}_{x}, \hat{\mathrm{V}}_{y}, \hat{\mathrm{V}}_{z}, \mathrm{C}_{\mathrm{h}}, \mathrm{C}_{\mathrm{v}}$ are unknown extrinsic calibration constant.s.

Thus; every point $P$ for which both 3D and image coordinates can be established, provides a linear equation involving 7 camera parameters (assuming $\mathrm{C}_{\mathrm{h}}=1$ ). A minimum of 7 points with known coordinates are, therefore, needed to solve for the system parameters. In reality, more than 7 points are used to overdetermine the problem and then solved by the least squares method. The remaining calibration parameters are solved by simple mathematical steps (refer section 2.2). This solution of the calibration parameters for a non-coplanar distribution. of object points in 3D is called 3D calibration.

One of the main reasons that the least squares solution may not exist for the system of linear equations in (3) above, is that the points used for calibration actually lie in a plane with respect to the world coordinate system. In this case, the calibration parameters are solved in two steps:

(1) First a basic set of parameters are solved by the least squares method from equation (4) below.

(2) Next the remaining parameters are solved by a set of simple mathematical steps as shown in Section 2.3.

This solution of the calibration parameters for a coplanar distribution of object points is referred to as $2 \mathrm{D}$ calibration. 
In this study we have assumed that it is the $z$-coordinate that is unimportant for 2D calibration. Equation (3) can be written without the $z$ components as follows:

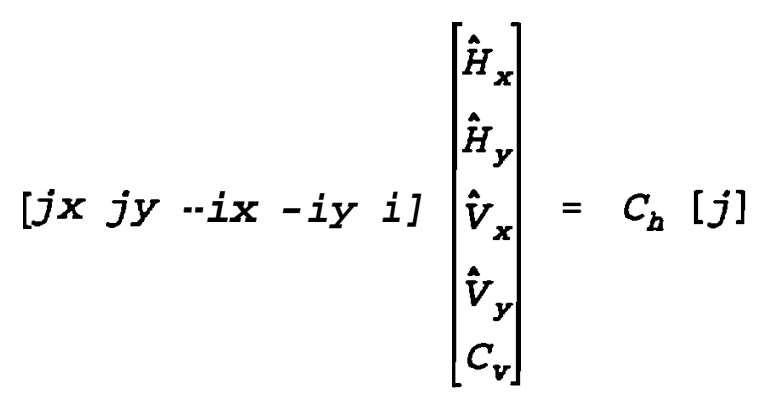

If the least squares solution does not exist in this case, it is then recommended that another set of points with more $x-y$ spread be selected for calibration purposes.

\subsection{Homogeneous Form for Coordinate Conversion:}

In this section we shall present a one-step transformation between the world and image coordinates. Although equations (1) and (2) are non-linear, it is possible to represent them in a condensed matrix linear transformation form by use of homogeneous coordinates. Within this context, the world to image coordinate transformation for 3D calibration case can be expressed as:

$$
k\left[\begin{array}{l}
i \\
j \\
1
\end{array}\right]=\left[\begin{array}{llll}
\hat{H}_{x} & \hat{H}_{y} & \hat{H}_{z} & -C_{b} \\
\hat{V}_{x} & \hat{V}_{y} & \hat{V}_{z} & -C_{\mathrm{v}} \\
\frac{\hat{A}_{x}}{f} & \frac{\hat{A}_{y}}{f} & \frac{\hat{A}_{z}}{f} & -\frac{C_{\mathrm{a}}}{f}
\end{array}\right]\left[\begin{array}{l}
x \\
y \\
z \\
1
\end{array}\right]
$$

Likewise the coordinate conversion for 2D calibration case can be expressed in matrix form as: 


$$
k\left[\begin{array}{l}
i \\
j \\
1
\end{array}\right]=\left[\begin{array}{lll}
\hat{H}_{x} & \hat{H}_{y} & -C_{b} \\
\hat{V}_{x} & \hat{V}_{y} & -C_{v} \\
\frac{\hat{A}_{x}}{f} & \frac{\hat{A}_{y}}{f} & -\frac{C_{a}}{f}
\end{array}\right]\left[\begin{array}{l}
x \\
y \\
1
\end{array}\right]
$$

In general, the $3 \times 4$ matrix in $3 D$ case (equation 5 ) and $3 \times 3$ matrix in $2 \mathrm{D}$ case (equation 6) that relate the homogeneous forms of the image and world coordinates are called the camera

matrices. A $3 \times 3$ orthonormal matrix $R$ (refer equation 29) can be obtained from the camera matrix in the 3D case. The correspondence between world and image coordinates is obtained by a complete solution of all entries of the camera matrices as shown in Sections 2.2 and 2.3 .

\subsection{Computation of the Calibration Parameters - 3D Case:}

For the 3D calibration case, assuming $\mathbf{C}_{\boldsymbol{h}}=1$, we can compute camera garameters $\hat{\mathbf{H}}_{\mathbf{x}}, \hat{\mathrm{H}}_{\mathrm{y}}, \hat{\mathbf{H}}_{\mathrm{z}}, \hat{\mathbf{V}}_{\mathbf{x}}, \hat{\mathbf{V}}_{\mathbf{y}}, \hat{\mathbf{V}}_{\mathrm{z}}$ and $\mathbf{C}_{\mathbf{v}}$ from equation (3). The remaining entries of the camera matrix (refer equation 5) $\mathbf{C}_{h}$, $\hat{A}_{x} / f, \hat{A}_{y} / f, \hat{A}_{z} / \mathbf{f}$ and $C_{\mathbf{z}} / \mathbf{f}$ can be computed by the following steps:

(1) From the property $\mathbf{I}$ Hi $=\mathbf{I}=1$ of the camera coordinate vectors $\hat{\mathbf{H}}$ and $\hat{\mathbf{v}}$, we can compute parameter $\boldsymbol{c}_{\mathbf{b}}$ by the fol.lowing equations:

$$
\left|C_{h}\right|=\sqrt{\frac{1}{\hat{H}_{x}^{2}+\hat{H}_{y}^{2}+\hat{H}_{z}^{2}}}
$$

or

$$
\left|c_{h}\right|=\sqrt{\frac{1}{\hat{V}_{x}^{2}+\hat{V}_{y}^{2}+\hat{V}_{z}^{2}}}
$$

(2) Assuming the sign of $\mathrm{C}$, as +1 , update values of $\hat{\mathrm{H}}_{\mathrm{x}}, \hat{\mathrm{H}}_{\mathrm{y}}, \hat{\mathrm{B}}_{\mathbf{z}}$, $\hat{V}_{x}, \hat{V}_{y}, \hat{V}_{z}$, and $C_{v}$ by multiplying them by $C_{b}$. The sign of parameter $\mathrm{C}$, can be obtained (from equations 1 and 2 ) from any object point $\mathbf{P}_{0}\left(\mathbf{x}_{0}, \mathbf{Y}_{0}, \mathbf{z}_{0}\right)$ whose measured image

Page 14 
coordinates are $\left(i_{0}, j_{0}\right)$ such that both $i_{0}$ and $j_{0}$ are not zeros:

$$
\begin{aligned}
& \operatorname{sign}\left(C_{b}\right)=\frac{\operatorname{sign}\left(x_{0} \hat{H}_{x}+y_{0} \hat{H}_{y}+z_{0} \hat{H}_{z}-C_{b}\right)}{\operatorname{sign}\left(\hat{i}_{0}\right)} \\
& \operatorname{or} \\
& \operatorname{sign}\left(C_{b}\right)=\frac{\operatorname{sign}\left(x_{0} \hat{V}_{x}+y_{0} \hat{V}_{y}+z_{0} \hat{V}_{z}-C_{v}\right)}{\operatorname{sign}\left(j_{0}\right)}
\end{aligned}
$$

The remaining parameters $\left(\hat{\mathrm{A}}_{x}, \hat{\mathrm{A}}_{y}, \hat{\mathrm{A}}_{2}, \mathrm{C}_{\mathrm{a}}, \mathrm{f}\right)$ can be solved by any one of the following two methods:

\section{Method I:}

(1) Parameters $\left(\hat{\mathrm{A}}_{x}, \hat{\mathrm{A}}_{y}, \hat{\mathrm{A}}_{z}\right)$ can be obtained from the right-handed property of the camera coordinate system $(B, V, A)$ as the cross product of vectors $\left(\hat{\mathrm{H}}_{\mathrm{x}}, \hat{\mathrm{H}}_{\mathrm{y}}, \hat{\mathrm{H}}_{\mathrm{z}}\right)$ and $\left(\hat{\mathrm{V}}_{\mathrm{x}}, \hat{\mathrm{V}}_{\mathrm{y}}, \hat{\mathrm{V}}_{\mathrm{z}}\right)$.

(2) Camera parameters $f$ and $C$ can be obtained from the following matrix equation derived from equations (1) and (2):

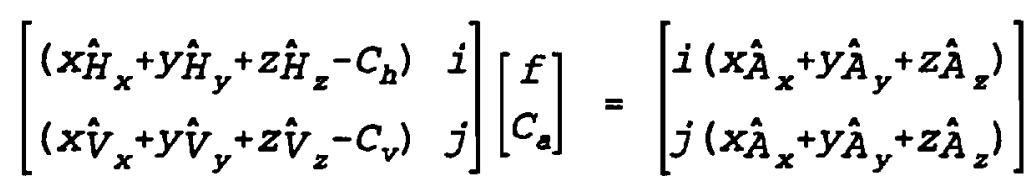

The above equation is solved from several calibration points $P$ whose image points $(i, j)$ are measured and object points $(\mathrm{X}, \mathrm{Y}, \mathbf{z})$ are known.

\section{Method II:}

(1) Solve for parameters $\hat{\mathrm{A}}_{x} / f, \hat{\mathrm{A}}_{y} / f, \hat{\mathrm{A}}_{z} / f$ and $C_{\mathrm{z}} / f$ as follows from known values of $\hat{B}, \hat{v}, C_{h}$ and $C_{v}$ computed before. 


$$
\left[\begin{array}{cccc}
i x & i y & i z & -i \\
j x & j y & j z & -j
\end{array}\right]\left[\begin{array}{c}
\frac{\hat{A}_{x}}{f} \\
\frac{\hat{A}_{y}}{f} \\
\frac{A_{z}}{f} \\
\frac{C_{a}}{f}
\end{array}\right]=\left[\begin{array}{l}
\left(x \hat{H}_{x}+y \hat{H}_{y}+z \hat{H}_{z}-C_{b}\right) \\
\left(x \hat{V}_{x}+y \hat{V}_{y}+z \hat{V}_{z}-C_{v}\right)
\end{array}\right] \ldots
$$

(2) The focal length $f$ can be computed from the property $\mid \mathbb{A}=1$ as shown below:

$$
f^{2}=\frac{1}{\left(\frac{\hat{A}_{x}}{f}\right)^{2}+\left(\frac{\hat{A}_{y}}{f}\right)^{2}+\left(\frac{\hat{A}_{z}}{f}\right)^{2}}
$$

The parameters computed by the above algorithm without imposing any constraint on the least squares solution may not lead to an orthonormal rotation matrix $R$. In order to obtain an orthonormal solution for $R$ in the $3 D$ case, the least squares solution can be modified as shown in Appendix $I$.

\subsection{Computation of the Calibration Parameters - 2D Case:}

The parameter computation in the 2D case (refer camera matrix in equation 6 ) is more complex due to the fact that we can solve a smaller subset of camera parameters from the linear equation (4). We need to compute the remaining camera parameters, namely $\hat{A}_{x} / f, \hat{A}_{y} / f, C_{a} / f$ and $C_{h}$ in the following steps:

(1) Assuming $C_{h}=1$, compute parameters $\hat{A}_{x} / f, \hat{A}_{y} / f$ and $C_{a} / f$ from the following matrix equation derived from equations (1) and (2): 


$$
\left[\begin{array}{ccc}
i x & i y & -i \\
j x & j y & -j
\end{array}\right]\left[\begin{array}{c}
\hat{A}_{x} \\
f \\
\frac{\hat{A}_{y}}{f} \\
\frac{C_{a}}{f}
\end{array}\right]=\left[\begin{array}{c}
\left(x \hat{H}_{x}+y \hat{H}_{y}-I\right) \\
\left(x \hat{V}_{x}+y \hat{V}_{y}-C_{v}\right)
\end{array}\right] \ldots
$$

Equation (8) is solved from several points $\mathrm{P}$ whose image points $(i, j)$ are measured and object points $(x, y)$ are known.

(2) Assuming $\mathbf{C}_{\mathbf{h}}=1$, the focal length f can be obtained from the orthonormal property of the rotation matrix $R$ (equation 29) by the equation below. An explanation for the derivation of parameter $f$ is given in Appendix II.

$$
f^{2}=-\left(\frac{\hat{H}_{x} \hat{H}_{y}+\hat{V}_{x} \hat{V}_{y}}{\frac{\hat{A}_{x}}{f} \frac{\hat{A}_{y}}{f}}\right)
$$

(3) Parameter $\boldsymbol{C}_{\boldsymbol{h}}$ can be obtained from the orthonormal property of rotation matrix $R$ by the following equations. .A derivation of these equations is shown in Appendix II.

$$
\left|C_{h}\right|=\sqrt{\frac{1}{\hat{H}_{x}^{2}+\hat{V}_{x}^{2}+\left(\frac{\hat{A}_{x}}{f}\right)^{2} f^{2}}}
$$

or

$$
\left|C_{h}\right|=\sqrt{\frac{1}{\hat{H}_{y}^{2}+\hat{V}_{y}^{2}+\left(\frac{\hat{A}_{y}}{f}\right)^{2} f^{2}}}
$$

(4) Assuming the sign of $\mathbf{C}_{b}$ as +1 , update values of $\hat{\mathbf{H}}_{\mathbf{x}}, \hat{\mathbf{H}}_{\mathbf{y}}, \hat{\mathbf{V}}_{\mathbf{x}}$, $\hat{\mathbf{v}}_{y}, C_{\nabla}, \hat{\mathrm{A}}_{\mathbf{z}} / \mathbf{f}, \hat{\mathrm{A}}_{\mathrm{y}} / \mathbf{f}$ and $\mathrm{C}_{\mathbf{a}} / \mathbf{f}$ by multiplying them with $\mathbf{C}_{\mathrm{h}}$. Compute the sign of $C_{h}$ from any object point $P_{0}\left(x_{0}, Y_{0}\right)$ in $3 D$ 
whose measured image coordinates are $\left(i_{0}, j_{0}\right)$ such that both $i_{0}$ and $j_{0}$ are not zeros:

$$
\operatorname{sign}\left(C_{h}\right)=\frac{\operatorname{sign}\left(x_{0} \hat{H}_{x}+y_{0} \hat{H}_{y}-C_{h}\right)}{\operatorname{sign}\left(\hat{i}_{0}\right)}
$$

or

$$
\operatorname{sign}\left(C_{h}\right)=\frac{\operatorname{sign}\left(x_{0} \hat{V}_{x}+y_{0} \hat{V}_{y}-C_{v}\right)}{\operatorname{sign}\left(j_{0}\right)}
$$

(5) With the correct sign of $C_{b}$, update values of $\hat{H}_{x}, \hat{H}_{y}, V_{x}, \hat{V}_{y}$, $C_{v}, \hat{A}_{x} / f, \hat{A}_{y} / f$ and $C_{a} / f$ for the camera matrix in equation $(6)$.

\subsection{Robustness of the Calibration Algorithm}

The final goal of most camera calibration algorithms is the robust and accurate computation of image and world coordinates. In most state of the art calibration methods, some int.rinsic parameters such as image center displacement and scale! factor have sicjnificant influence on these computations. In this section, we shall analyze the effects of these intrinsic parameters on the extrinsic parameters computed by our algorithm, and also on the computation of image and world coordinates. A strong robustness of our parameter computation procedure will be demonstrated in this section.

In most camera calibration methods, the center of the image or sensor plane is chosen as the image center $S$ (refer Figure 1). The actual center of the image plane is the intersection of the optical axis of the camera-lens system with the image plane. Due to scanning and sampling errors or due to inaccuracies; in the lens system, the actual image center can be different from the center $\mathbf{S}$ of the image coordinate system(I-J). Another intrinsic parameter commonly considered in camera calibration is the scale factor $\left(s_{i}, s_{j}\right)$. The scale factor parameter is caused by the dimension, spacing and number of sensor elements in the camera 
sensor, and also by the scanning and sampling properties of the analog-to-digital converter of the image processor. In $[13,18]$ different methods of computing these parameters have been discussed.

Let us consider a point in 3D whose image coordinates are $\left(i_{f}, j_{f}\right)$ with respect to the center $S$ (refer Figure 1 ) of the image coordinate system (I-J). Let the actual center of the image plane be at location $\left(i_{0}, j_{0}\right)$ with respect to $s$. Let $(i, j)$ be the true location of the point in the image plane with respect to its actual image center. If the scale factor is $\left(s_{1}, s_{j}\right)$ we can obtain the following expressions $[13,18,23]$ :

$$
\begin{aligned}
& i=s_{i}^{-1}\left(i_{f}-i_{0}\right) \\
& j=s_{j}^{-1}\left(j_{f}-j_{0}\right)
\end{aligned}
$$

If the camera is calibrated with image coordinates $\left(i_{f}, j_{f}\right)$, we shall obtain extrinsic parameters $\hat{\mathrm{H}}^{\prime}, \hat{\mathrm{V}}^{\prime}, \hat{\mathrm{A}}^{\prime}, \mathrm{C}_{\mathrm{h}}{ }^{\prime}, \mathrm{C}_{\mathrm{v}}{ }^{\prime}$, and $\mathrm{C}^{\prime}$. Let $\hat{H}, \hat{V}, \hat{A}, C_{h}, C_{v}$, and $C$ be the calibration parameters with no lens distortion, no image center displacement and scale factor. From equations (1) and (2) the combined effects of these intrinsic parameters on the extrinsic parameters as follows:

$$
\begin{aligned}
& \hat{H}^{\prime}=s_{j} \hat{H}+i_{0} \frac{\hat{A}}{f} \\
& \hat{V}^{\prime}=s_{j} \hat{V}+j_{0} \frac{\ddot{A}}{f} \\
& C_{h}{ }^{\prime}=s_{i} C_{h}+i_{0} \frac{C_{a}}{f} \\
& C_{v}{ }^{\prime}=s_{j} C_{v}+j_{0} \frac{C_{a}}{f}
\end{aligned}
$$

In Method I of the 3D case, the axial vector $\hat{A}$ is expressed (refer Section 2.2) as follows: 


$$
\hat{A}^{\prime}=\hat{H}^{\prime} \times \hat{V}^{\prime}=s_{i} s_{j} \hat{A}-s_{i} j_{0} \frac{\hat{V}}{f}-s_{j} i_{0} \frac{\hat{H}}{f}
$$

This error in the axial vector $\hat{A}^{\prime}$ from its ideal value $\hat{A}$ will produce an error in the computation of image or world coordinates in a 3D stereo setup.

For Method II of the 3D case, and for the 2D calibration case, from equations (7) and (8) respectively, we shall obtain the foil-owing expressions.

$$
\begin{aligned}
& \frac{\hat{A}^{\prime}}{f^{\prime}}=\frac{\hat{A}}{f} \\
& \frac{C_{a}^{\prime}}{f^{\prime}}=\frac{C_{a}}{f}
\end{aligned}
$$

From these expressions (equations 12 and 14) we obtain the following theorems:

\section{Theorem 1:}

In Method II of the 3D case and the 2D calibration case, there $i$ is no error in the computation of image coordinates $\left(i_{f}, j_{f}\right)$ from world coordinates $(x, y, z)$ if the parameters used for computation are $\hat{\mathrm{H}}^{\prime}, \hat{\mathrm{V}}^{\prime}, \hat{\mathrm{A}}^{\prime}, \mathrm{C}_{\mathrm{b}}{ }^{\prime}, \mathrm{C}_{\mathrm{v}}{ }^{\prime}$, and $\mathrm{C}_{\mathrm{a}}{ }^{\prime}$.

\section{Theorem 2:}

In Methods II of the 3D case and the 2D calibration case, there $i$ s no error in the computation of world coordinates $(x, y, z)$ from uncompensated image coordinates $\left(i_{f}, j_{f}\right)$ with parameters $\hat{\mathrm{H}}^{\prime}$, $\hat{\mathrm{V}}^{\prime}, \hat{\mathrm{A}}^{\prime}, \mathrm{C}_{\mathrm{b}}{ }^{\prime}, \mathrm{C}_{\mathrm{\nabla}}{ }^{\prime}$, and $\mathrm{C}_{\mathrm{a}}{ }^{\prime}$.

Proofs of Theorems 1 and 2 are given in Appendix III. These theorems prove a strong robust property of the proposed calibration algorithm which implies that we can obtain accurate image and world coordinates without extensive computation of image center displacement and scale factor. In cases where accurate calibration parameters are needed, equations (12)-(14) 
can be used to update the extrinsic parameters for different values of image center displacements and scale factors. Experimental results (Section 8 ) are provided to verify the analysis presented in this section.

\section{4:.0 Calibration Model With Complete Lens Distortion}

In this section, we shall consider the effects of lens distortion on the proposed calibration model for both $3 D$ and $2 D$ cases. Figure 1 shows the ideal image pixel coordinates $(i, j)$ of a point $\mathrm{P}$ in 3D. However, due to lens distortions, the actual coordinates of the image point may be at $\left(i_{p}, j_{p}\right)$. This change in the pixel coordinates due to lens distortion leads to a variation of the scale of an image as a function of position in the image plane. Clearly, for accurate calibration of the camera., it is important to correct for distortions in the lens. When image center displacement and scale factor are added to the calibration model, the expressions of $(i, j)$ in equation (11) are used for $\left(i_{p}, j_{p}\right)$

Two types of lens distortions commonly seen in image processing applications are radial[5,16] and tangential[4,16] distortions. Two types of common radial distortions in image processing are pincushion and barrel distortions shown in Figure 2. In the simplest case, as for third order or Siedel distortion[16], an outward displacement of a given image point from its desired location on a mean image plane is referred to as pincushion distortion. An inward displacement, on the other hand, is called barrel distortion. Besides these, there can be tangential distortions usually caused by: (a) decentering of the lens (Decentering Distortion), or (b) imperfections in lens manufacturing or tilt in camera sensor or lens (Thin Prism Distortion).

One commonly used model for correcting lens distortion is that developed by Brown $[4,5,16]$. Let $D_{1}$ and $D_{j}$ be the corrections for geometric lens distortions present in the image coordinates $i_{p}$ and $j_{F^{\prime}}$ respectively of an object point $P(x, y, z)$. With 
$r=\left(i_{p}{ }^{2}+j_{F}{ }^{2}\right)^{1 / 2}, D_{i}$ and $D_{j}$ are expressed by the following infinite series:

$$
\begin{aligned}
& D_{1}=i_{p}\left(l_{-1} r^{2}+l_{2} r^{4}+l_{3} r^{6}+\ldots\right)+\left(p_{1}\left(r^{2}+2 i_{p}{ }^{2}\right)+2 p_{2} i_{p} j_{p}\right)\left(1+p_{3} r^{2}+\ldots\right) \\
& D_{j}=j_{p}\left(l_{-1} r^{2}+l_{2} r^{4}+l_{3} r^{6}+\ldots\right)+\left(2 p_{1} i_{p} j_{p}+p_{2}\left(r^{2}+2 j_{p}^{2}\right)\right)\left(1+p_{3} r^{2}+\ldots\right) \ldots
\end{aligned}
$$

The coefficients $l_{1}, l_{2}, l_{3}, p_{1}, p_{2}$ and $p_{3}$ may be determined as a part of the camera calibration process. The model accounts for both symmetric radial and asymmetric tangential distortions. The terms including coefficients $l_{1}, l_{\text {, and }} l_{3}$ represent radial distortion, and the terms which include $p_{1}, p_{2}$ and $p_{3}$ represent tangential distortions. The image coordinates are corrected for lens distortion by the following expression:

$$
\begin{aligned}
& i=i_{p}+D_{i} \\
& j=j_{p}+D_{j}
\end{aligned}
$$

The calibration equations under lens distortion can be obtained. by substituting $\left(i_{p}+D_{i}\right)$ for $i$ and $\left(j_{p}+D_{j}\right)$ for $j$ in equation. (3) for the $3 D$ case and equation (4) for the 2D case. The solution for the unknown camera parameters $\left(\hat{\mathrm{H}}_{x}, \hat{\mathrm{H}}_{y}, \hat{\mathrm{H}}_{z}, \hat{\mathrm{V}}_{x}, \hat{\mathrm{V}}_{\mathrm{y}}, \hat{\mathrm{V}}_{z}, \mathrm{C}_{\mathrm{h}}, \mathrm{C}_{\mathrm{v}}\right)$ and the lens distortion parameters $\left(l_{1}, 1_{2}, 1_{3}, p_{1}, p_{2}, p_{3}\right)$ are obtained by using the method of lagrange multipliers[14] for a constrained least squares solution. The remaining camera parameters $\left(\hat{\mathrm{A}}_{x}, \hat{\mathrm{A}}_{y}, \hat{\mathrm{A}}_{\mathrm{z}}, \mathrm{C}_{\mathrm{a}}, \mathrm{f}\right)$ can be obtained by the steps mentioned before in Sections 2.2 and 2.3.

In the following two sections we shall discuss a new improved calibration algorithm under (a) radial and (b) both radial and tangential lens distortions by a constrained least squares solution with Lagrange multipliers.

\subsection{Calibration Under Radial Lens Distortion}

Let us consider a vector $B$ for the unknown extrinsic calibration parameters in the $3 D$ calibration case as follows:

$$
B^{T}=\left[\begin{array}{lllllll}
\hat{H}_{x} & \hat{H}_{y} & \hat{H}_{z} & \hat{V}_{x} & \hat{V}_{y} & \hat{V}_{z} & C_{v}
\end{array}\right]
$$


Let $U$ and $W$ be two vectors with known image and world coordinate values a.s defined below:

$$
\begin{aligned}
& U_{n}^{T}=\left[j_{n} x_{n} j_{n} Y_{n} j_{n} z_{n}-i_{n} x_{n}-i_{n} Y_{n}-i_{n} z_{n} i_{n}\right] \\
& W_{a}^{T}=r_{a}^{2} U_{n}^{T} \\
& \text { where } r_{n}^{2}=\left(i_{n}{ }^{2}+j_{n}{ }^{2}\right) .
\end{aligned}
$$

Also define variable $a_{1}=-j_{n} r_{n}{ }^{2}$. Subscript $n$ represents the $n^{\text {th }}$ calibration point $(n=1, \ldots, N)$ used for this algorithm.

Assuming $C_{h}=1$, we can represent the linear equation under radial lens distortion as follows:

$$
\left[\begin{array}{lll}
B^{T} & C^{T} & l_{1}
\end{array}\right]\left[\begin{array}{l}
U_{n} \\
W_{n} \\
a_{n}
\end{array}\right]=j_{n}, \quad n=1, \ldots, N
$$

The constraint is: $C^{T}=I_{1} B^{T}$.

Solving the above linear equation by least squares we shall obtain the following expressions for $B$ and 1 ,.

$$
\begin{aligned}
& B=\left[\sum_{n=1}^{N}\left(U_{n}+I_{1} W_{n}\right)\left(U_{n}+I_{1} W_{n}\right) T\right]^{-1}\left[\sum_{n=1}^{N}\left(j_{n}-I_{1} a_{n}\right)\left(U_{n}+I_{1} W_{n}\right)\right] \\
& I_{1}=\frac{\sum_{n=1}^{N} \frac{\left(j_{n}-B^{T} U_{n}\right)\left(B^{T} W_{n}+a_{n}\right)}{\sum_{n=1}^{N}\left|\left(B^{T} W_{n}+a_{n}\right)\right|^{2}}}{\ldots(19)}
\end{aligned}
$$

\section{Iterative Algorithm For Accurate Solution of $B$ and $\underline{1}_{1}$ :}

This one-step solution for the extrinsic parameters in vector $B$ and the radial lens distortion parameter 1 , can be further improved by an iterative procedure described in Steps 1-4 below:

Step 1: Choose an initial "reasonable" value of 1 , (say 0.1 ). 
Step 2: Compute extrinsic parameter vector B by equation (18).

Step 3: Compute radial distortion parameter 1, by equation (19) with the value of vector $B$ obtained in Step 2.

Step 4: Repeat steps 2 and 3 till a maximum number of iterations.

The number of iterations required for this computation depends on the starting value of 1 . However, we have proven analytically that the above algorithm converges towards a correct solution of 1 , in consecutive steps of iteration.

\section{Proof of Conversence of the Iterative Solution of $B$ and $1_{1}$ :}

We shall use the symbols $e_{k}, B_{k}$, and 1 for the values of least squared error, extrinsic parameter vector $B$, and radial distortion parameter 1 , respectively in the $k^{\text {th }}$ step of iteration. Following these notations:

$$
\begin{aligned}
& e^{2}{ }_{k+1}=\sum_{n=1}^{N}\left(j_{n}-B^{T}{ }_{k+1}\left(U_{n}+1_{k+1} W_{n}\right)-1_{k+1} a_{n}\right)^{2} \\
& \leq \sum_{n=1}^{N}\left(j_{n}-B_{k}{ }^{T}\left(U_{n}+I_{k+1} W_{n}\right)-I_{k+1} a_{n}\right)^{2} \\
& =\sum_{n=1}^{N}\left(\left(j_{n}-B_{k}^{T}\left(U_{n}+1_{k} W_{n}\right)-1_{k} a_{n}\right)+\left(1_{k}-1_{k+1}\right)\left(B_{k}{ }^{T} W_{n}+a_{n}\right)\right)^{2} \\
& =\left.\sum_{n=1}^{N}\left|\left(j_{n}-B_{k}{ }^{T}\left(U_{n}+1_{k} W_{n}\right)-1_{k} a_{n}\right) \|^{2}+\sum_{n=1}^{N}\left(1_{k}-1_{k+1}\right)^{2}\right|\left(B_{k}{ }^{T} W_{n}+a_{n}\right)\right|^{2} \\
& +2 \sum_{n=1}^{N}\left(1_{k}-1_{k+1}\right)\left(j_{n}-B_{k}{ }^{T}\left(U_{n}+I_{k} W_{n}\right)-1_{k} a_{n}\right)\left(B_{k}{ }^{T} W_{n}+a_{n}\right)
\end{aligned}
$$




$$
\begin{aligned}
&=e_{k}^{2}+\sum_{n=1}^{N}\left[2\left(I_{k}-I_{k+1}\right)\left(j_{n}-B_{k}^{T} U_{n}\right)\left(B_{k}^{T} W_{n}+a_{n}\right)+\left(I_{k+1}^{2}-I_{k}^{2}\right)\left|B_{k}^{T} W_{n}+a_{n}\right|^{2}\right] \\
&=e_{k}^{2}+\sum_{n=1}^{N} \mid B_{k}^{T} W_{n}+a_{n} \|^{2}\left[I_{k+1}^{2}+2\left(I_{k}-1_{k+1}\right) \frac{\left(j_{n}-B_{n}^{T} U_{n}\right)\left(B_{k}^{T} W_{n}+a_{n}\right)}{\left|B_{k}^{T} W_{n}+a_{n}\right|^{2}}-I_{k}^{2}\right] \\
&=e_{k}^{2}+\sum_{n=1}^{N}\left|B_{k}^{T} W_{n}+a_{n} \|^{2}\right|\left(I_{k+1}-\frac{\left(j_{n}-B_{k}^{T} U_{n}\right)\left(B_{k}^{T} W_{n}+a_{n}\right)}{\mid B_{k}^{T} W_{n}+a_{n} \|^{2}}\right)^{2}- \\
&\left.\left(I_{k}-\frac{\left(j_{n}-B_{k}^{T} U_{n}\right)\left(B_{k}^{T} W_{n}+a_{n}\right)}{\left|B_{k}^{T} W_{n}+a_{n}\right|^{2}}\right)^{2}\right]
\end{aligned}
$$

Considering the terms within the square brackets as a function of $\mathbf{I}_{\mathbf{k}+1}$, this function reaches a minimum at

$$
I_{k+1}=-\frac{\left(j_{n}-B_{k}{ }^{T} U_{n}\right)\left(B_{k}{ }^{T} W_{n}+a_{n}\right)}{\left\|B_{k}^{T} W_{n}+a_{n}\right\|^{2}}
$$

The resulting value of the function at the minimum is

$$
-\left(l_{k}-\frac{\left(j_{n}-B_{k}^{T} U_{n}\right)\left(B_{n}^{T} W_{n}+a_{n}\right)}{\left|B_{n}^{T} W_{n}+a_{n}\right|^{2}}\right)^{2}
$$

which cannot be positive.

From this we can infer

$$
e_{k+1}^{2} \leq e_{k}^{2}
$$

The experimental analysis in Section 8 for parameter 1 , support the fast convergence of the above algorithm. For our experimental setup, starting from $\boldsymbol{1}_{\mathbf{1}}=0.1$, we converged at $\boldsymbol{1}_{\mathbf{1}}=-$ 0.000052 in just one iteration. Further iterations do not change the value of 1 , significantly (less than 1\%). 


\subsection{Calibration Under Radial and Tanqential Lens Distortions}

The analysis for the radial distortion can be extended to both radial and tangential lens distortions. For this we shall consider vectors $Q$ and $S$ as follows:

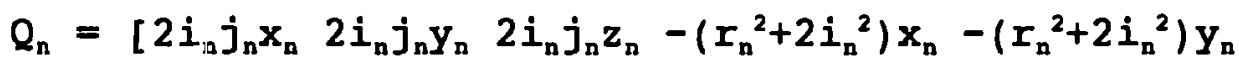
$\left.-\left(r_{n}^{2}+2 i_{n}^{2}\right) z_{n}\left(r_{n}^{2}+2 i_{n}^{2}\right)\right]$

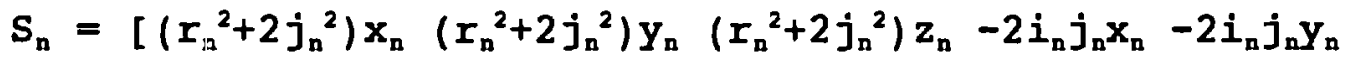

$$
\left.-2 i_{n} j_{n} z_{n} 2 i_{n} j_{n}\right]
$$

Also define variables $b$ and $c$ as follows:

$b_{n}=-2 i_{n} j_{n}$

$c_{n}=-\left(r_{13}^{2}+2 j_{n}^{2}\right)$

As before, subscript $\mathrm{n}$ is for the $\mathrm{n}^{\text {th }}$ calibration point $(n=1, \ldots, N)$.

The linear equation under both radial and tangential lens distortions can be expressed as below:

$$
\left[\begin{array}{lllllllll}
B^{T} & C^{T} & D^{T} & E^{T} & I_{1} & p_{1} & p_{2}
\end{array}\right]\left[\begin{array}{c}
U_{n} \\
W_{n} \\
Q_{n} \\
S_{n} \\
a_{n} \\
b_{n} \\
C_{n}
\end{array}\right]=j_{n}, \quad n=1, \ldots, N
$$

where the constraints are:

$$
C^{T}=l_{1} B^{T}, D^{T}=P_{1} B^{T}, E^{T}=P_{2} B^{T}
$$

Solving the above linear equation by least squares we shall obtain the following expressions for vector $B$ and variables $l_{1}$, $p_{1 I}$ and $p_{2}$. 


$$
B=\left[\sum_{n=1}^{N} z_{n} z_{n}^{T}\right]^{-1} \sum_{n=1}^{N}\left(j_{n}-\left(l_{1} a_{n}+p_{1} b_{n}+p_{2} c_{n}\right)\right) z_{n} \quad \ldots .
$$

where $\mathbf{z}_{n}=\left(U_{n}+l_{1} W_{n}+p_{1} Q_{n}+p_{2} S_{n}\right)$

$$
\begin{aligned}
& I_{1}=\frac{\sum_{n=1}^{N}\left(\left(j_{n}-B^{T}\left(U_{n}+p_{1} Q_{n}+p_{2} S_{n}\right)-\left(p_{1} b_{n}+p_{2} C_{n}\right)\right)\left(B^{T} W_{n}+a_{n}\right)\right.}{\sum_{n=1}^{N}\left|B^{T} W_{n}+a_{n}\right|^{2}} \ldots \\
& p_{1}=\frac{\sum_{n=1}^{N}\left(\left(j_{n}-B^{T}\left(U_{n}+I_{1} W_{n}+p_{2} S_{n}\right)-\left(I_{1} a_{n}+p_{2} C_{n}\right)\right)\left(B^{T} Q_{n}+b_{n}\right)\right.}{\sum_{n=1}^{N}\left|B^{T} Q_{n}+b_{n}\right|^{2}} \ldots \\
& p_{2}=\frac{\sum_{n=1}^{N}\left(\left(j_{n}-B^{T}\left(U_{n}+I_{1} W_{n}+p_{1} Q_{n}\right)-\left(I_{1} a_{n}+p_{1} b_{n}\right)\right)\left(B^{T} S_{n}+C_{n}\right)\right.}{\sum_{n=1}^{N}\left|B^{T} S_{n}+C_{n}\right|^{2}} \ldots
\end{aligned}
$$

These first estimates for these parameters can be further improved by extending the iterative procedure discussed in Section 4.1 to the radial and tangential case. Extensive experiments were conducted for this case. Our experimental results suggest that the convergence of this algorithm depends very much on the starting values of $l_{1}, p$, and $p$, Unlike the radial distortion case, improper choices of the starting values lead to solutions at local minima and do not produce the correct estimates for the extrinsic parameters in vector B. The details of the experimental results are in Section 8 . 


\subsection{Complete Calibration Algorithm}

In this section, we shall extend the robust property of our calibration method and the new solution for the lens distortion parameters, to create a new algorithm for the solution of all calibration parameters. This algorithm has the combined strengths of all the procedures discussed before. An outline of this algorithm is shown in Figure 3.

The proposed complete algorithm partitions the parameters into two subsets:

(1) image center displacement and scale factor, and

(2) lens distortion, focal length and the extrinsic parameters. The first step of the algorithm solves the first subset of parameters by state of the art procedures[13,23] assuming the second subset of parameters as constant.

In the second step of the algorithm, the second subset of parameters are computed by the procedure in section 4 . In this step, the image center displacement and scale factor are assumed constant. Since only a subset of the extrinsic parameters are solved first, the remaining extrinsic parameters are computed by the steps outlined in Sections 2.2 and 2.3. The orthonormality of the rotation matrix $R$ is determined by the procedure in Appendix I.

The two-step procedure to determine a complete solution of all calibration parameters leads to a robust solution. In this algorithm, we have partitioned the parameter space in a way that is different from state of the art methods[25] for the solution of all calibration parameters. We submit that our algorithm leads to a more robust solution. The reasons for this robustness are as follows :

(1) We have demonstrated in Section 3, the effects of image center displacement and scale factor on the extrinsic parameters (equations 12-14). These effects are mostly linear (equation 12) or no effect at all (equation 14). The effect of lens distortion parameters, on the other hand, is 
more complex and can not be determined in a closed form like equations $(12)-(14)$.

(2) The effects of image center displacement and scale factor is same for all pixels in an image, whereas, the lens distortion effect is different for different pixels.

The state of the art procedure in [25] partitions the parameter space with the lens distortion parameters as a separate subset from the remaining parameters. We submit that due to the close and complex link between the lens distortion parameters and the extrinsic parameters, the algorithm in [25] may converge to a local minimum leading to inaccurate results. Also any analytical proof of convergence of this algorithm has not been provided. In our algorithm, on the other hand, we have computed the lens distortion and extrinsic parameters in a single step.

\subsection{Error Analysis}

In this section we shall consider the effects of measurement errors in image coordinates $(i, j)$ on the computation of the camera calibration parameters. Let us consider errors $e_{1}$ and $e_{2}$ in the measurement of image coordinates $i$ and $j$ respectively. Ignoring effects of second order error terms, the errors in extrinsic parameters are proportional to the following terms:

$e_{1} \sum j x, \quad e_{2} \sum j y, \quad e_{1} \sum j z, \quad e_{1} \sum j, \quad e_{2} \sum i x, e_{2} \sum i y, e_{2} \sum i z$, $e_{2} \sum j x, e_{2} \sum j y, e_{2} \sum j z, e_{2} \sum i$

where $\Sigma$ is for all calibration points.

This result shows us that the errors in the calibration parameters are proportional to the $x-y-z$ spread of the calibration points, and the $i-j$ spread of the image coordinates of the calibration points. The errors also decrease as the origin $W$ (refer Figure 1 ) of the world coordinate system is closer to the center of the field of view. Thus the errors in the calibration parameters are less when the camera is calibrated 
over a narrow field of view.

It i.s clear from equation (1) that errors in parameters $\hat{\theta}$ and $C_{h}$ have most effect on the computation of image coordinate $i$. From equation (2) we can determine that errors in parameters $\hat{v}$ and $C_{v}$ have most effects on the computation of the image coordinate $j$. Errors in the computation of world coordinates is a complex function of errors in all the calibration parameters.

\subsection{Incremental Model}

In many machine vision applications, such as defect inspection and part size measurement, it is not necessary to use the calibration model and the parameter computation algorithms for complete solution of all camera parameters discussed before. In such applications, we may only need to calibrate distances in the image plane to distances or tolerances in the object world. For such applications, we frequently need to check if an image feature such as a defect or a part edge is within a tolerance or not. These tolerances are usually specified in real-world units whereas the measurements are performed in the image domain. It is therefore sufficient to establish a correspondence between tolerances in 3D to distances in image (or sensor) plane.

The equations describing the correspondence between the tolerances can be obtained from the homogeneous expressions in equations (5) and (6). Let $\left(x_{1}, y_{1}, z_{1}\right)$ and $\left(x_{2}, Y_{2}, z_{2}\right)$ be two points in $3 D$ with corresponding image coordinates $\left(i, j_{1}\right)$ and $\left(i_{1}, j_{2}\right)$ respectively. Let $\left(\Delta x=x_{1}-x_{2}, \Delta y=y_{1}-y_{2}, \Delta z=z_{1}-z_{2}\right)$ be tolerances in $3 D$ and $\left(\Delta i=i_{1}-i_{2}, \Delta j=j_{1}-j_{2}\right)$ be tolerances in the image coordinates. The correspondence equations between tolerances for the 3D case are as follows: 
$k_{1}\left[\begin{array}{l}\Delta i \\ \Delta j\end{array}\right]=\left[\begin{array}{l}\left(\hat{H}_{x}-i_{2} \frac{\hat{A}_{x}}{f}\right)\left(\hat{H}_{y}-i_{2} \frac{\hat{A}_{y}}{f}\right)\left(\hat{H}_{z}-i_{2} \frac{\hat{A}_{z}}{f}\right) \\ \left(\hat{V}_{x}-j_{2} \frac{\hat{A}_{x}}{f}\right)\left(\hat{V}_{y}-j_{2} \frac{\hat{A}_{y}}{f}\right)\left(\hat{V}_{z}-\hat{j}_{2} \frac{\hat{A}_{z}}{f}\right)\end{array}\right]\left[\begin{array}{l}\Delta x \\ \Delta y \\ \Delta z\end{array}\right]$

or

$$
k_{2}\left[\begin{array}{l}
\Delta i \\
\Delta j
\end{array}\right]=\left[\begin{array}{l}
\left(\hat{H}_{x}-i_{1} \frac{\hat{A}_{x}}{f}\right)\left(\hat{H}_{y}-i_{1} \frac{\hat{A}_{y}}{f}\right)\left(\hat{H}_{z}-i_{1} \frac{\hat{A}_{z}}{f}\right) \\
\left(\hat{V}_{x}-j_{1} \frac{\hat{A}_{x}}{f}\right)\left(\hat{V}_{y}-j_{1} \frac{\hat{A}_{y}}{f}\right)\left(\hat{V}_{z}-j_{1} \frac{\hat{A}_{z}}{f}\right)
\end{array}\right]\left[\begin{array}{l}
\Delta x \\
\Delta y \\
\Delta z
\end{array}\right]
$$

where

$$
k_{t}=\frac{\left(x_{t} \hat{A}_{x}+y_{t} \hat{A}_{y}+z_{t} \hat{A}_{z}-C_{a}\right)}{f}, \quad 1 \leq t \leq 2
$$

Similarly for the 2D calibration case, the correspondence between the two tolerances can be represented as below:

$$
k_{1}\left[\begin{array}{l}
\Delta i \\
\Delta j
\end{array}\right]=\left[\begin{array}{l}
\left(\hat{H}_{x}-i_{2} \frac{\hat{A}_{x}}{f}\right)\left(\hat{H}_{y}-i_{2} \frac{\hat{A}_{y}}{f}\right) \\
\left(\hat{V}_{x}-j_{2} \frac{\hat{A}_{x}}{f}\right)\left(\hat{V}_{y}-j_{2} \frac{\hat{A}_{y}}{f}\right)
\end{array}\right]\left[\begin{array}{l}
\Delta x \\
\Delta y
\end{array}\right]
$$

or

$$
k_{2}\left[\begin{array}{l}
\Delta i \\
\Delta j
\end{array}\right]=\left[\begin{array}{l}
\left(\hat{H}_{x}-i_{1} \frac{\hat{A}_{x}}{f}\right)\left(\hat{H}_{y}-i_{1} \frac{\hat{A}_{y}}{f}\right) \\
\left(\hat{V}_{x}-j_{1} \frac{\hat{A}_{x}}{f}\right)\left(\hat{V}_{y}-j_{1} \frac{\hat{A}_{y}}{f}\right)
\end{array}\right]\left[\begin{array}{l}
\Delta x \\
\Delta y
\end{array}\right]
$$

where 


$$
k_{t}=\frac{\left(x_{t} \hat{A}_{x}+y_{t} \hat{A}_{y}-C_{a}\right)}{f}, \quad 1 \leq t \leq 2
$$

or

$$
k_{t}=-\frac{\hat{H}_{z} C_{b}+\hat{V}_{z} C_{v}+\hat{A}_{z} C_{q}}{\hat{H}_{z} i_{t}+\hat{V}_{z} j_{t}+\hat{A}_{z} f}, \quad 1 \leq t \leq 2
$$

The incremental models in equations (25) and (26) can be used in applications where we need to convert tolerances in the object world to tolerances in the image plane or vice-versa. The 2D solution in equation (26) can be used in its entirety in many applications such as defect size measurement, where we! can covert the size! exactly to real-world dimensions by using equation (26) if the location of the defect in the image is known.

We can further simplify the correspondence equations, in both $2 D$ and 3D cases, by considering a "nominal" value $\left(i_{0}, j_{0}\right)$ for the image coordinates $\left(i, j_{1}\right)$ or $\left(i, j_{2}\right)$. An example of such a "nominal" value is the center of the image or sensor plane. By this assumption, the entries of the transformation matrices in equatio $s$ (25) and (26) are constants that can be computed by the least squares approach from several (at least 3 ) known values of object tolerances $((\Delta x, \Delta y, \Delta z)$ in $3 D$ case and $(\Delta x, \Delta y)$ in $2 D$ case) and measured values of image tolerances $(\Delta i, \Delta j)$.

When lens distortion, displacement of image center and scale factor are added to the calibration model, equations (25) and (26) can be extended by using the expression for $i$ and $j$ in equations (11) and (16). An example of these correspondence equations with image center displacement only is provided below. Let $\left(i_{0}, j_{0}\right)$ be the image center displacements for image points $\left(i_{1}, j_{1}\right)$ and $\left(i_{2}, j_{2}\right)$. The correspondence equations for the $3 D$ case are as follows: 
$k_{1}\left[\begin{array}{l}\Delta i \\ \Delta j\end{array}\right]=\left[\begin{array}{l}\left(\hat{H}_{x}-\left(i_{2}-i_{0}\right) \frac{\hat{A}_{x}}{f}\right)\left(\hat{H}_{y}-\left(i_{2}-i_{0}\right) \frac{\hat{A}_{y}}{f}\right)\left(\hat{H}_{z}-\left(i_{2}-i_{0}\right) \frac{\hat{A}_{z}}{f}\right) \\ \left(\hat{V}_{x}-\left(j_{2}-j_{0}\right) \frac{\hat{A}_{x}}{f}\right)\left(\hat{V}_{y}-\left(j_{2}-j_{0}\right) \frac{\hat{A}_{y}}{f}\right)\left(\hat{V}_{z}-\left(j_{2}-j_{0}\right) \frac{\hat{A}_{z}}{f}\right)\end{array}\right]\left[\begin{array}{l}\Delta x \\ \Delta y \\ \Delta z\end{array}\right]$

or

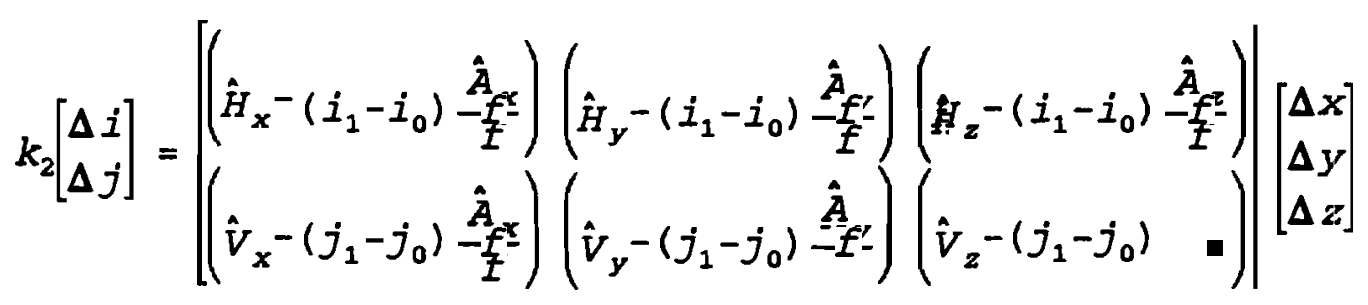

$k_{1}, k_{2}$ are defined before in equation (25).

For the 2D case the corresponding equations are as follows:

$$
k_{1}\left[\begin{array}{l}
\Delta i \\
\Delta j
\end{array}\right]=\left[\begin{array}{l}
\left(\hat{H}_{x}-\left(i_{2}-i_{0}\right) \frac{\hat{A}_{x}}{f}\right)\left(\hat{H}_{y}-\left(i_{2}-i_{0}\right) \frac{\hat{A}_{y}}{f}\right) \\
\left(\hat{V}_{x}-\left(j_{2}-j_{0}\right) \frac{\hat{A}_{x}}{f}\right)\left(\hat{V}_{y}-\left(j_{2}-j_{0}\right) \frac{\hat{A}_{y}}{f}\right)
\end{array}\right]\left[\begin{array}{l}
\Delta x \\
\Delta y
\end{array}\right]
$$

or

$$
k_{2}\left[\begin{array}{l}
\Delta i \\
\Delta j
\end{array}\right]=\left[\begin{array}{l}
\left(\hat{H}_{x}-\left(i_{1}-i_{0}\right) \frac{\hat{A}_{x}}{f}\right)\left(\hat{H}_{y}-\left(i_{1}-i_{0}\right) \frac{\hat{A}_{y}}{f}\right) \\
\left(\hat{V}_{x}-\left(j_{1}-j_{0}\right) \frac{\hat{A}_{x}}{f}\right)\left(\hat{V}_{y}-\left(j_{1}-j_{0}\right) \frac{\hat{A}_{y}}{f}\right)
\end{array}\right]\left[\begin{array}{l}
\Delta x \\
\Delta y
\end{array}\right] \ldots(28)
$$

$k_{1}, k_{2}$ are defined before in equation (26). 


\subsection{Experimental Results}

In this section we shall apply the proposed algorithms on a test setup and analyze the results of these experiments. The experiments were conducted for a coplanar set of object points and the calibration parameters were obtained.

\subsection{Experimental Setup:}

The test calibration points were created by accurately placing a set of 100 holes one inch apart on a precisely polished metal plate in a square grid of 10 dots horizontally and 10 dots vertically. The image of the test calibration points was acquired by a NEC TI-23A camera[17] manufactured by NEC Corporation and a Fujinon $50 \mathrm{~mm}$ focal length TV lens. The camera was positioned at an angle of approximately 20 degrees from vertical with the calibration plate placed on a horizontal surface. The images were processed by an image processing hardware manufactured by Applied Intelligent systems Inc[1]. The image resolution was $512 \times 480$ pixels. The centroid pixel of each dot was obtained to extract the pixel coordinates of the object points. Figure 4 shows a picture of the calibration setup.

\subsection{Test Results for Extrinsic Parameters:}

In the test setup, the image coordinates of the calibration points, with known world coordinates, were measured from the acquired image. The 2D calibration algorithm (refer Section 2) was applied to the coplanar set of test points to obtain the extrinsic calibration parameters. The accuracy of the calibration algorithm is presented in two steps:

(1) The difference between the actual world coordinates and the computed world coordinates of the test points.

(2) The difference between the measured image coordinates and the computed image coordinates of the test points.

In the first step, the extrinsic parameters were obtained 
(Section 2.3) from known world coordinates and measured image coordinates of the test calibration points. A new image was acquired and the world coordinates of the calibration points were computed from the measured image coordinates and the estimated calibration parameters. The computed and actual world coordinates of the test calibration points are reported in Table 2. The experiment was repeated for all 100 test points.

In the second step, the image coordinates of the test points were obtained from the estimated calibration parameters and the actual world coordinates. The measured and the computed image coordinates are reported in Table 3. This experiment was also repeated for all 100 test points.

TABLE 2. True and Computed World Coordinates of the Calibration Points.

$$
\begin{aligned}
& \left(\hat{H}_{x}=0.780698, \quad \hat{H}_{y}=0.153695, \quad C_{b}=3.320078,\right. \\
& \hat{V}_{x}=-0.287919, \hat{V}_{y}=0.994583, \quad C_{v}=-0.651146, \\
& \left.\hat{A}_{x} / f=-0.000166, \hat{A}_{y} / f=-0.000040, C_{a} / f=-0.053424\right)
\end{aligned}
$$

\begin{tabular}{crrrrrr} 
Test & True Coords & \multicolumn{2}{c}{ Computed Coords } & \multicolumn{2}{c}{ Errors } \\
Points & $\mathrm{X}$ & $\mathrm{Y}$ & $\mathrm{X}$ & $\mathrm{Y}$ & $\mathrm{X}$ & $\mathrm{Y}$ \\
1 & 0 & 0 & 0.0115 & -0.0068 & 0.0115 & 0.0068 \\
2 & 0 & 1 & 0.0189 & 1.0146 & 0.0189 & 0.0146 \\
3 & 0 & 2 & 0.0263 & 2.0343 & 0.0263 & 0.0343 \\
4 & 0 & 3 & 0.0436 & 3.0018 & 0.0436 & 0.0018 \\
5 & 0 & 4 & -0.0145 & 4.0004 & 0.0145 & 0.0004 \\
6 & 1 & 0 & 0.9774 & 0.0031 & 0.0226 & 0.0031 \\
7 & 1 & 1 & 0.9940 & 0.9708 & 0.0060 & 0.0292 \\
8 & 1 & 2 & 1.0006 & 1.9875 & 0.0006 & 0.0125 \\
9 & 1 & 3 & 1.0071 & 3.0027 & 0.0071 & 0.0027 \\
10 & 1 & 4 & 1.0236 & 3.9657 & 0.0236 & 0.0343 \\
11 & 2 & 0 & 2.0123 & -0.0188 & 0.0123 & 0.0188 \\
12 & 2 & 1 & 2.0181 & 0.9963 & 0.0181 & 0.0037 \\
13 & 2 & 2 & 1.9591 & 1.9914 & 0.0409 & 0.0086 \\
14 & 2 & 3 & 1.9649 & 3.0035 & 0.0351 & 0.0035 \\
15 & 2 & 4 & 1.9707 & 4.0140 & 0.0293 & 0.0140 \\
16 & 3 & 0 & 2.9662 & -0.0089 & 0.0338 & 0.0089 \\
17 & 3 & 1 & 2.9614 & 1.0534 & 0.0386 & 0.0534
\end{tabular}




$\begin{array}{lllllll}18 & 3 & 2 & 2.9763 & 2.0136 & 0.0237 & 0.0136 \\ 19 & 3 & 3 & 2.9813 & 3.0224 & 0.0187 & 0.0224 \\ 20 & 3 & 4 & 2.9961 & 3.9795 & 0.0039 & 0.0205 \\ 21 & 4 & 0 & 3.9785 & 0.0196 & 0.0215 & 0.0196 \\ 22 & 4 & 1 & 3.9827 & 1.0283 & 0.0173 & 0.0283 \\ 23 & 4 & 2 & 3.9869 & 2.0355 & 0.0131 & 0.0355 \\ 24 & 4 & 3 & 3.9911 & 3.0411 & 0.0089 & 0.0411 \\ 25 & 4 & 4 & 4.0052 & 3.9952 & 0.0052 & 0.0048 \\ 26 & 5 & 0 & 4.9942 & -0.0022 & 0.0058 & 0.0022 \\ 27 & 5 & 1 & 4.9878 & 1.0534 & 0.0122 & 0.0534 \\ 28 & 5 & 2 & 5.0010 & 2.0074 & 0.0010 & 0.0074 \\ 29 & 5 & 3 & 5.0044 & 3.0099 & 0.0044 & 0.0099 \\ 30 & 5 & 4 & 5.0078 & 4.0108 & 0.0078 & 0.0108 \\ 31 & 6 & 0 & 6.0034 & -0.0238 & 0.0034 & 0.0238 \\ 32 & 6 & 1 & 6.0061 & 0.9786 & 0.0061 & 0.0214 \\ 33 & 6 & 2 & 6.0087 & 1.9795 & 0.0087 & 0.0205 \\ 34 & 6 & 3 & 6.0113 & 2.9788 & 0.0113 & 0.0212 \\ 35 & 6 & 4 & 6.0139 & 3.9765 & 0.0139 & 0.0235 \\ 36 & 7 & 0 & 7.0063 & -0.0452 & 0.0063 & 0.0452 \\ 37 & 7 & 1 & 6.9983 & 1.0037 & 0.0017 & 0.0037 \\ 38 & 7 & 2 & 7.0002 & 2.0014 & 0.0002 & 0.0014 \\ 39 & 7 & 3 & 7.0118 & 2.9479 & 0.0118 & 0.0521 \\ 40 & 7 & 4 & 7.0038 & 3.9921 & 0.0038 & 0.0079 \\ \text { Average } & & & & & 0.0157 & 0.0187 \\ \text { Standard } & \text { Deviation } & & & 0.0115 & 0.0146\end{array}$

TABLE 3. Measured and Computed Image Coordinates of the Calibration Points.

$\left(\hat{\mathrm{H}}_{x}, \hat{\mathrm{H}}_{y}, \mathrm{C}_{\mathrm{h}}, \hat{\mathrm{V}}_{\mathrm{x}}, \hat{\mathrm{V}}_{\mathrm{y}}, \mathrm{C}_{\mathrm{v}}, \hat{\mathrm{A}}_{\mathrm{x}} / \mathrm{f}, \hat{\mathrm{A}}_{\mathrm{y}} / \mathrm{f}, \mathrm{C}_{\mathrm{a}} / \mathrm{f}\right.$ same as Table 2)

Test Meas Coords

Points $I \quad J$

$1 \quad-62 \quad 12$

$2-59 \quad 31$

$3-56 \quad 50$

$4-53 \quad 68$
Computed Coords

I

$-62.1460$

$-59.3138$

$-56.4772$

$-53.6364$
$\mathrm{J}$

12.1883

30.8283

49.4965

68.1927
Errors

I

0.1460

0.1883

0.3138

0.1717

0.4772

0.5035

0.6364

0.1927 


\begin{tabular}{|c|c|c|c|c|c|c|}
\hline 5 & -51 & 87 & -50.7913 & 86.9172 & 0.2087 & 0.0828 \\
\hline 6 & -48 & 7 & -47.6809 & 6.8202 & 0.3191 & 0.1798 \\
\hline 7 & -45 & 25 & -44.8288 & 25.5143 & 0.1712 & 0.5143 \\
\hline 8 & -42 & 44 & -41.9725 & 44.2366 & 0.0275 & 0.2366 \\
\hline 9 & -39 & 63 & -39.1118 & 62.9873 & 0.1118 & 0.0127 \\
\hline 10 & -36 & 81 & -36.2468 & 81.5663 & 0.2468 & 0.5663 \\
\hline 11 & -33 & 1 & -33.1252 & 1.4184 & 0.1252 & 0.4184 \\
\hline 12 & -30 & 20 & -30.2533 & 20.1669 & 0.2533 & 0.1669 \\
\hline 13 & -28 & 39 & -27.3769 & 38.9438 & 0.6231 & 0.0562 \\
\hline 14 & -25 & 58 & -24.4962 & 57.7492 & 0.5038 & 0.2508 \\
\hline 15 & -22 & 77 & -21.6111 & 76.5832 & 0.3889 & 0.4168 \\
\hline 16 & -19 & -4 & -18.4784 & -4.0171 & 0.5216 & 0.0171 \\
\hline 17 & -16 & 16 & -15.5862 & 15.6860 & 0.4138 & 0.3140 \\
\hline 18 & -13 & 34 & -12.6897 & 33.6178 & 0.3103 & 0.3822 \\
\hline 19 & -10 & 53 & -9.7888 & 52.4783 & 0.2112 & 0.5217 \\
\hline 20 & -7 & 71 & -6.8834 & 71.3674 & 0.1166 & 0.3674 \\
\hline 21 & -4 & -9 & -3.7393 & -9.4869 & 0.2607 & 0.4869 \\
\hline 22 & -1 & 10 & -0.8269 & 9.3713 & 0.1731 & 0.6287 \\
\hline 23 & 2 & 29 & 2.0900 & 28.2583 & 0.0900 & 0.7417 \\
\hline 24 & 5 & 48 & 5.0114 & 47.1741 & 0.0114 & 0.8259 \\
\hline 25 & 8 & 66 & 7.9372 & 66.1188 & 0.0628 & 0.1188 \\
\hline 26 & 11 & -15 & 11.0927 & -14.9912 & 0.0927 & 0.0088 \\
\hline 27 & 14 & 5 & 14.0257 & 4.7223 & 0.0257 & 0.2777 \\
\hline 28 & 17 & 23 & 16.9632 & 22.8648 & 0.0368 & 0.1352 \\
\hline 29 & 20 & 42 & 19.9052 & 41.8363 & 0.0948 & 0.1637 \\
\hline 30 & 23 & 61 & 22.8517 & 60.8369 & 0.1483 & 0.1631 \\
\hline 31 & 26 & -21 & 26.0186 & -20.5303 & 0.0186 & 0.4697 \\
\hline 32 & 29 & -2 & 28.9723 & -1.5611 & 0.0277 & 0.4389 \\
\hline 33 & 32 & 17 & 31.9306 & 17.4372 & 0.0694 & 0.4372 \\
\hline 34 & 35 & 36 & 34.8934 & 36.4647 & 0.1066 & 0.4647 \\
\hline 35 & 38 & 55 & 37.8608 & 55.5214 & 0.1392 & 0.5214 \\
\hline 36 & 41 & -27 & 41.0393 & -26.1046 & 0.0393 & 0.8954 \\
\hline 37 & 44 & -7 & 44.0140 & -7.0795 & 0.0140 & 0.0795 \\
\hline 38 & 47 & 12 & 46.9933 & 11.9750 & 0.0067 & 0.0250 \\
\hline 39 & 50 & 30 & 49.9771 & 30.0589 & 0.0229 & 0.0589 \\
\hline 40 & 53 & 50 & 52.9656 & 50.1722 & 0.0344 & 0.1722 \\
\hline \multicolumn{5}{|c|}{ Average } & 0.2140 & 0.3271 \\
\hline Sta & rd & eviat & & & 0.1811 & 0.2270 \\
\hline
\end{tabular}


We can see from Table 2 that the average errors in both $X$ and $Y$ coordinates are less than $2 \%$ of the unit distance between two adjacent test points. From Table 3 we see that the average error between the measured and computed pixel coordinates is less than 0.5 pixel. This error not only includes errors due to the calibration algorithm but also includes measurement and quantization errors. Both these results demonstrate the high accuracy of the calibration algorithm in Section 2.

\subsection{Test Results for Imase Center Displacement}

In this experiment the center $S$ (refer Figure 1 ) of the image coordinate system is displaced by a range of values and the extrinsic parameters computed by the 2D algorithm. For $i_{0}=j_{0}=$ 0 , the center $S$ of the image coordinate system is at the center of the digitized image $i . e$. at $(256,240)$ for a $512 \times 480$ resolution image. All subsequent values of $\left(i_{0}, j_{0}\right)$ are displacements of $s$ from this position. Also in this experiment we have assumed scale factor $s_{1}=s_{j}=1$ and lens distortion $D_{i}=D_{j}=0$.

Table 4 shows many different values of extrinsic parameters $\hat{A}_{x}^{\prime} / f^{\prime}, \hat{A}_{y}^{\prime} / f^{\prime}$ and $C_{a}^{\prime} / f^{\prime}$ (refer equation 14) for different displacements $\left(i_{0}, j_{0}\right)$ of the center $S$. It is clear from Table 4 that for several displacements of $S$, the parameters $\hat{A}_{x}^{\prime} / f^{\prime}, \hat{A}_{y}^{\prime} / f^{\prime}$ and $C_{a}^{\prime} / f^{\prime}$ have not changed much. This small variation .in these parameters support equation (14).

TABLE 4. Values of Parameters $\hat{A}_{x} / f, \hat{A}_{y} / f$ and $C_{a} / f$ for Different Displacements of Center $S$ of the Image coordinate System.

$\left(\hat{A}_{x} / f=-0.000158, \quad \hat{A}_{y} / f=-0.000124, \quad C_{a} / f=-0.052898\right)$

\begin{tabular}{||c|c|c|c|c||}
\hline$i_{0}$ & $j_{0}$ & $\hat{A}_{x}^{\prime} / f^{\prime}$ & $\hat{A}_{y}^{\prime} / f^{\prime}$ & $C_{a}^{\prime} / f^{\prime}$ \\
\hline 10 & 0 & -0.000148 & -0.000125 & -0.052819 \\
\hline 0 & 10 & $-0,000174$ & -0.000116 & -0.053063 \\
\hline 10 & 10 & -0.000167 & -0.000129 & -0.053031 \\
\hline
\end{tabular}




\begin{tabular}{|c|c|c|c|c|}
\hline 20 & 0 & -0.000141 & -0.000115 & -10.052732 \\
\hline 0 & 20 & -0.000178 & -0.000110 & -0.053151 \\
\hline 20 & 20 & -0.000172 & -0.000136 & -0.053129 \\
\hline 30 & 0 & -0.000139 & -0.000099 & -0.052673 \\
\hline 0 & 30 & -0.000172 & -0.000112 & -0.053181 \\
\hline 30 & 30 & -0.000178 & -0.000141 & -0.053173 \\
\hline 40 & 0 & -0.000141 & -0.000079 & -0.052637 \\
\hline 0 & 40 & -0.000167 & -0.000113 & -0.053226 \\
\hline 40 & 40 & -0.000200 & -0.000131 & -0.053172 \\
\hline 50 & 0 & -0.000145 & -0.000051 & -0.052581 \\
\hline 0 & 50 & -0.000165 & -0.000103 & -0.053296 \\
\hline 50 & 50 & -0.000236 & -0.000096 & -0.053137 \\
\hline 60 & 0 & -0.000149 & -0.000013 & -0.052463 \\
\hline 0 & 60 & -0.000165 & -0.000076 & -0.053384 \\
\hline 60 & 60 & -0.000242 & -0.000110 & -0.053200 \\
\hline 70 & 70 & -0.000239 & -0.000137 & -0.053272 \\
\hline Average & & -0.000174 & -0.000106 & -0.053011 \\
\hline Std. Dev. & & 0.000032 & 0.000031 & 0.000269 \\
\hline \hline
\end{tabular}

Table 5 summarizes the computed and adjusted values of parameters $\hat{\mathrm{H}}_{x}{ }^{\prime}, \hat{\mathrm{H}}_{y}{ }^{\prime}$, and $C^{\prime}$ for the same experiment. The adjusted values of these parameters are obtained from equation (12). $\hat{\mathrm{H}}_{\mathrm{x}} \mathrm{Adj}, \hat{\mathrm{B}}_{\mathrm{y}} \mathrm{Adj}$, and $\mathrm{C}_{\mathrm{h}} \mathrm{Adj}$ represent the adjusted value of parameters $\hat{H}_{x}^{\prime}, \hat{H}_{y}^{\prime}$, and $C_{b}{ }^{\prime}$ respectively. For example, for $i_{0}=40$ and $j_{0}=40, C^{\prime}$ $=5.158965$ and $C_{h}$ Adj $=C_{b}+i_{0}\left(C_{a} / f\right)=3.027859+(-40)(-.0530109)$ $=5.148295$. The adjusted value has an error $C_{h} E r r=0.01067$ from the computed value. The adjusted values of these parameters are very close to their experimental values, thus supporting equation 
(12). Table 6 shows the computed and adjusted values of parameters $\hat{V}_{x}, \hat{V}_{y}$, and $C$. These results also support equation (12).

TABLE 5. Computed and Adjusted Values of Parameters $\hat{\mathrm{B}}_{\mathbf{x}}, \hat{\mathrm{B}}_{\mathbf{y}}, \mathrm{C}_{\mathrm{b}}$ for Different Displacements of the Center $S$ of the Image Coordinate System.

$$
\begin{aligned}
& \left(\hat{\mathrm{B}}_{\mathrm{x}}=0.7623, \quad \hat{\mathrm{B}}_{\mathrm{y}}=0.1912, \quad \mathrm{C}_{\mathrm{b}}=3.0279\right. \text {, } \\
& \hat{\mathrm{A}}_{x} / f=-0.000174, \hat{\mathrm{A}}_{y} / f=-0.000106, C_{a} / f=-0.053011 \text { ) }
\end{aligned}
$$

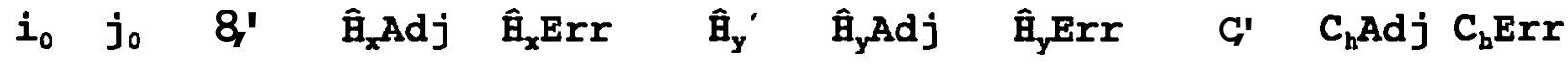

$\begin{array}{rrrllllllll}10 & 0 & 0.7632 & 0.7640 & 0.0008 & 0.1906 & 0.1923 & 0.0017 & 3.547 & 3.558 & 0.011 \\ 0 & 10 & 0.7641 & 0.7623 & 0.0018 & 0.1952 & 0.1912 & 0.0039 & 3.047 & 3.028 & 0.020 \\ 10 & 10 & 0.7650 & 0.7640 & 0.0010 & 0.1947 & 0.1923 & 0.0024 & 3.568 & 3.558 & 0.010 \\ 20 & 0 & 0.7644 & 0.7658 & 0.0014 & 0.1904 & 0.1933 & 0.0030 & 4.067 & 4.088 & 0.021 \\ 0 & 20 & 0.7656 & 0.7623 & 0.0033 & 0.1973 & 0.1912 & 0.0061 & 3.058 & 3.028 & 0.031 \\ 20 & 20 & 0.7676 & 0.7658 & 0.0018 & 0.1975 & 0.1933 & 0.0041 & 4.105 & 4.088 & 0.017 \\ 30 & 0 & 0.7659 & 0.7675 & 0.0016 & 0.1908 & 0.1944 & 0.0036 & 4.591 & 4.618 & 0.027 \\ 0 & 30 & 0.7663 & 0.7623 & 0.0040 & 0.1975 & 0.1912 & 0.0063 & 3.059 & 3.028 & 0.031 \\ 30 & 30 & 0.7694 & 0.7675 & 0.0019 & 0.1991 & 0.1944 & 0.0047 & 4.637 & 4.618 & 0.019 \\ 40 & 0 & 0.7676 & 0.7692 & 0.0016 & 0.1915 & 0.1955 & 0.0040 & 5.118 & 5.148 & 0.030 \\ 0 & 40 & 0.7668 & 0.7623 & 0.0045 & 0.1977 & 0.1912 & 0.0064 & 3.058 & 3.028 & 0.030 \\ 40 & 40 & 0.7699 & 0.7692 & 0.0007 & 0.1977 & 0.1955 & 0.0023 & 5.159 & 5.148 & 0.011 \\ 50 & 0 & 0.7692 & 0.7710 & 0.0018 & 0.1915 & 0.1965 & 0.0050 & 5.643 & 5.678 & 0.036 \\ 0 & 50 & 0.7675 & 0.7623 & 0.0052 & 0.1987 & 0.1912 & 0.0075 & 3.058 & 3.028 & 0.031 \\ 50 & 50 & 0.7698 & 0.7710 & 0.0012 & 0.1927 & 0.1965 & 0.0038 & 5.673 & 5.678 & 0.005 \\ 60 & 0 & 0.7699 & 0.7727 & 0.0028 & 0.1899 & 0.1976 & 0.0077 & 6.159 & 6.209 & 0.050 \\ 0 & 60 & 0.7683 & 0.7623 & 0.0061 & 0.2005 & 0.1912 & 0.0093 & 3.060 & 3.028 & 0.033 \\ 60 & 60 & 0.7728 & 0.7727 & 0.0000 & 0.1944 & 0.1976 & 0.0032 & 6.209 & 6.209 & 0.000 \\ 70 & 70 & 0.7760 & 0.7745 & 0.0015 & 0.1980 & 0.1986 & 0.0006 & 6.749 & 6.739 & 0.011 \\ \text { Average } & & 0.0021 & & & 0.0043 & & & 0.021 \\ \text { Standard Deviation } & 0.0017 & & & 0.0024 & & & 0.013\end{array}$


TABLE 6. Computed and Adjusted values of Parameters $\hat{v}_{x}, \hat{V}_{y}, C_{v}$ for Different Displacements of the Center $S$ of the Image Coordinate System.

$$
\begin{aligned}
& \left(\hat{v}_{x}=-0.3454, \quad \hat{v}_{y}=0.9575, \quad C_{v}=-1.2592\right. \text {, } \\
& \hat{\mathrm{A}}_{\mathrm{x}} / \mathrm{f}=-0.000174, \hat{\mathrm{A}}_{y} / f=-0.000106, \mathrm{C}_{\mathrm{a}} / \mathrm{f}=-0.053011 \text { ) }
\end{aligned}
$$

\begin{tabular}{|c|c|c|c|c|c|c|c|c|c|c|}
\hline & 0 & & & & & & & & & \\
\hline & 10 & -.3445 & -0.3437 & 18 & 9597 & 9586 & 00 & -0.735 & 29 & \\
\hline & 10 & -.3440 & -0. & 13 & & & & & & \\
\hline & 0 & & & & & & & & & \\
\hline & 20 & & & & & & & & & \\
\hline & 20 & - & & & & & & & & . \\
\hline & 0 & 9 & - & & & & & & & .00 \\
\hline & 30 &.- & - & & & & & & & .00 \\
\hline & 30 & 3 & 2 & & & & & & & .00 \\
\hline & 0 & 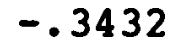 & - & & & & & & & 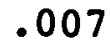 \\
\hline 0 & 40 & 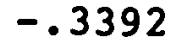 & - & & & & & & & 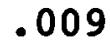 \\
\hline & 40 & -.33 & -0 & & & & & & & .00 \\
\hline & 0 & -.34 & -0 & 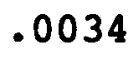 & 74 & & 01 & 50 & 59 & .00 \\
\hline 0 & 50 & -.33 & -0 & .0 & 18 & & 50 & 07 & 91 & .01 \\
\hline & 50 & -.33 & -0 . & .0 & 18 & & 50 & & & 00 \\
\hline & 0 & 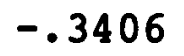 & -0 & .0 & 9 & & 03 & 46 & 59 & .01 \\
\hline 0 & 60 & 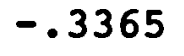 & -0 & .0 & 6 & & 57 & & & .02 \\
\hline & 50 & 3314 & -0.3 &. & 9697 & & & & & .00 \\
\hline & 0 & 8 & 3 & & & & & 64 & & 01 \\
\hline & & & & & & & & & & 60 \\
\hline & & & & .001 & & & .0022 & & & 00 \\
\hline
\end{tabular}

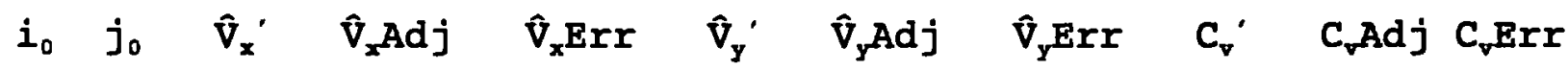

\subsection{Test. Results for Lens Distortion}

In order to generate reasonable distortions of the lens, we chose a $8 \mathrm{~mm}$ lens for our imaging setup. We used state of the art methods[13] for the computation of image center displacement and assumed that there is no scale factor distortion in the image. We then computed the calibration parameters without compensating for lens distortion and computed the errors in the image and world 
coordinate computations (shown by iterations-0 in Tables 7 and 8). Next we repeated this experiment with compensation for radial and tangential lens distortions by the proposed algorithm in Section 4.2. The average and standard deviation of errors in the image and world coordinate computations for various iterations of this algorithm are shown in Tables 7 and 8.

TABLE 7. Errors in the Computation of Image Coordinates for Different Iteration of the Lens Distortion Algorithm. Iteration-0 is for No Lens Distortion Compensation. Image Center Displacements are $i_{0}=6, j_{0}=4$.

\begin{tabular}{|c|c|c|c|c||}
\hline \hline Iterations & \multicolumn{2}{|c|}{ Errors (Average) } & \multicolumn{2}{c||}{ Errors (Std. Dev.) } \\
\hline & $\mathrm{I}$ & $\mathrm{J}$ & $\mathrm{I}$ & $\mathrm{J}$ \\
\hline 0 & 0.6530 & 0.4498 & 0.6096 & 0.3274 \\
\hline 1 & 0.4218 & 0.4496 & 0.3482 & 0.3592 \\
\hline 2 & 0.3574 & 0.4315 & 0.2812 & 0.3494 \\
\hline 3 & 0.3559 & 0.4167 & 0.2617 & 0.3393 \\
\hline 4 & 0.3547 & 0.4158 & 0.2613 & 0.3449 \\
\hline 5 & 0.3533 & 0.4156 & 0.2608 & 0.3414 \\
\hline
\end{tabular}

TABLE 8. Errors in the Computation of World Coordinates for Different Iteration of the Lens Distortion Algorithm. Iteration $=0$ is for No Lens Distortion Compensation. Image Center Displacements are $i_{0}=6, j_{0}=4$.

\begin{tabular}{|c|c|c|c|c|}
\hline \multirow[t]{2}{*}{ Iterations } & \multicolumn{2}{|c|}{ Errors (Average) } & \multicolumn{2}{|c|}{ Errors (Std. Dev.) } \\
\hline & $\mathbf{x}$ & $\mathbf{Y}$ & $\mathbf{x}$ & $\mathbf{Y}$ \\
\hline 0 & 0.0264 & 0.0147 & 0.0242 & 0.0108 \\
\hline 1 & 0.0170 & 0.0144 & 0.0138 & 0.0116 \\
\hline 2 & 0.0145 & 0.0138 & 0.0112 & 0.01 .12 \\
\hline
\end{tabular}




\begin{tabular}{||l|l|l|l|l||}
\hline 3 & 0.0145 & 0.0135 & 0.0133 & 0.0109 \\
\hline 4 & 0.0144 & 0.0135 & 0.0109 & 0.0110 \\
\hline 5 & 0.0144 & 0.0133 & 0.0104 & 0.0109 \\
\hline
\end{tabular}

It is clear from Tables 7 and 8 that the proposed algorithm for lens distortion compensation improves the calibration accuracy in the first two steps of iteration. Subsequent steps of iteration causes less than 1\% improvement in accuracy. As mentioned before in Section 4, the accuracy of this algorithm for both radial and tangential cases depend on the proper selection of the starting values of $l_{1}, p_{1}$, and $p_{1}$. In the radial case, however, the algorithm converges (within 3 iterations) to within $1 \%$ of the final value for different starting values of parameter $1_{1}$.

\subsection{Conclusions}

In this paper we have presented a new and simple algorithm for camera calibration based on state of the art geometrical models $[22,28]$. We have established a direct transformation between the 3D object world and the 2D image plane in terms of homogeneous vectors, and have computed the extrinsic camera parameters in simple steps by fast and accurate algorithms.

Next we have demonstrated a strong robust property of the proposed method, that if the camera is calibrated with, image data (not compensated for image center displacement and scale factor), our calibration algorithm yields parameters that produce no errors in the computation of image and world coordinates. This robust property of the proposed algorithm has been shown by both analytical and experimental means. This appears to be the first known analytical proof of its kind for robustness in a. calibration algorithm.

Furthermore, we have presented a new algorithm for the solution, of (a) radial and, (b) both radial and tangential lens 
distortions by a constrained least squares approach. An analytical proof for the convergence of this algorithm is also shown. Experimental results to support the algorithm and its convergence are presented. While other procedures exist for the solution of lens distortion parameters, we appear to have shown the only analytical proof of convergence for this algorithm.

Next, a complete algorithm for the solution of all calibration parameters is shown. The improved robustness of this new algorithm is discussed in light of state of the art procedures for the solution of all calibration parameters.

We have also presented the new Incremental Model for tolerances between the 3D world coordinates and the 2D sensor coordinates. All these analyses have been discussed for both coplanar and non-coplanar distributions of object points in 3D. Experimental results have been presented to support the calibration model and parameter computation algorithms.

All discussions on camera calibration presented in this paper can be extended to a multi-camera stereo setup.

\subsection{References}

[1] Applied Intelligent Systems Inc., "Hardware Installation and Reference Manual for AIS-3000, AIS-3500, and AIS-4000", August 22, 1990, Ann Arbor, Michigan.

[2] M.L. Baird, "Sight I: A computer vision system for automated IC chip manufacture", IEEE Trans. Syst. Man Cybern. 8, 2 (Feb) 1978, pp 133-139.

[3] J.R. Birk, R.B. Kelley and H. Martins, "An Orienting Robot for Feeding Workpieces stored in Bins", IEEE Trans. Syst. Man Cybern. 11, 2(Feb), 1981, pp 151-160.

[4] Duane C. Brown, "Decentering Distortion of Lenses", Photogrammetric Engineering, Vol 32, 1966, pp 444-462.

[5] Duane C. Brown, "Close-Range Camera Calibration", Photogrammetric Engineering, Vol 37, 1971, pp 855-866.

[6] John Cardillo and Maher A. Sid-Ahmed, "3D Position Sensing Using a Passive Monocular Vision System", IEEE Transactions 
on Pattern Analysis and Machine Intelligence, Vol. 13, No. 8, August 1991, pp. 809-813.

[7] K.S. Fu, "Robot vision for machine part recognition", Proceedings of the Society of Photo-Optical Instrumentation Engineers Conference on Robotics and Robot Sensing Systems (San Diego, California, August), Vol 442, SPIE, Bellingham, Washington.

[8] D.B. Gennery, "Stereo-Camera Calibration", Proceedings Image Understanding Workshop, November 1979, pp 101-108.

[9] S. Ganapaty, "Decomposition of Transformation Matrices for Robot Vision", in Proc. International Conference on Robotics and. Automation, 1984, pp 130-139.

[10] William I. Grosky and Louis A. Tamburino, "A Unified Approach to the Linear Camera Calibration Problem", IEEE Transactions on Pattern Analysis and Machine Intelligence, Vol. 12, No. 7, July 1990, pp. 663-671.

[11] Y.Y. Hsieh and K.S. Fu, "A method for automatic IC chip alignment and wire bonding", Proc. IEEE Computer Society Conf. on Patt. Recogn. and Image Proc. (Chicago, IL, Aug), IEEE, New York, pp 101-108.

[12] H. Itoh, A. Miyauchi, and S.Ozawa, "Distance measuring method using only simple vision constructed from moving robots", in Proc. 7th International Conference on Pattern Recognition, Montreal PQ Canada, Vol 1, 1984, pp 192.

[13] Rejmer K. Lenz and Roger Y. Tsai, "Techniques for Calibrating the Scale Factor and Image Center for High Accuracy 3-D Machine Vision Metrology", IEEE Transactions on Pattern Analysis and Machine Intelligence, Vol. 10, No. 5, September 1988, pp. 713-720.

[14] David Luenberger, "Linear and Non-Linear Programming", Addison-Wesley, Reading, Massachusetts, 1984.

[15] J.Y.S. Luh and J.A. Klaasen, "A Three-Dimensional.Vision by Off-Shelf System with Multi-Cameras", IEEE Transactions on Pattern Analysis and Machine Intelligence, Vol PAMI-7, No 1, January 1985, pp 35-45.

[16] Marual of Photogrammetry, 4th edition, American Society of Photogrammetry, 1980.

[17] NEC! Corporation, "TI-23A CCD Camera Instruction Manual", Tokyo, Japan. 
[18] Michael A. Penna, "Camera Calibration: A Quick and Easy Way to Determine the Scale Factor", IEEE Transactions on Pattern Analysis and Machine Intelligence, Vol. 13, No. 12, December 1991, pp. 1240-1245.

[19] W.A. Perkins, "A Model-Based Vision System for Industrial Parts", IEEE Trans. Comput. 27, 2(Feb), pp 126-143.

[20] A. Pugh, Ed. 1983, "Robot Vision", Springer-Verlag, New York.

[21] Irwin Sobel, "On Calibrating Computer Controlled Cameras for Perceiving 3-D Scenes", Artificial Intelligence, Vol 5, 1974, pp 185-198.

[22] Ivan E. Sutherland, "Three-Dimensional Data Input by Tablet", Proceedings of the IEEE, Vol 62, No 4, April 1974, pp 453-461.

[23] Roger Y. Tsai, "A Versatile Camera Calibration Technique for High-Accuracy 3D Machine Vision Metrology Using Off-theShelf TV Cameras and Lenses", IEEE Journal of Robotics and Automation, Vol RA-3, No 4, August 1987, pp 323-343.

[24] Ling-Ling Wang and Wen-Hsiang Tsai, "Camera Calibration by Vanishing Lines for 3D Computer Vision", IEEE Transactions on Pattern Analysis and Machine Intelligence, Vol. 13, No. 4, April 1991, pp. 370-376.

[25] Juyang Weng, et al, "Camera Calibration with Distortion Models and Accuracy Evaluation", IEEE Transactions on Pattern Analysis and Machine Intelligence, Vol14, No10, October 1992 .

[26] Prof. Dr. Ing. Wolfgang Faig, "Calibration of Close-Range Photogrammetric Systems: Mathematical Formulation", Photogrammetric Engineering and Remote Sensing, Vol 41, No 12, December 1975, pp 1479-1486.

[27] Dr. Kam w. Wong, "Mathematical Formulation and Digital Analysis in Close-Range Photogrammetry", Photogrammetric Engineering and Remote Sensing, Vol 41, No 11, November 1975, pp 1355-1375.

[28] Y. Yakimovsky and R. Cunningham, "A System for Extracting Three-Dimensional Measurements from a Stereo Pair of TV Cameras", Computer Graphics and Image Processing, Vol 7, April 1978, pp 195-210. 


\section{Appendix I}

\section{Algorithm for Parameters with Orthonormal $\mathbf{R}$ in the 3D (Non-Coplanar) Case}

The extrinsic calibration parameters computed in Section 2.2 can be improved to produce an orthonormal rotation matrix $R$ (equation 29) by the algorithm below obtained from a constrained least squares solution with Lagrange multipliers.

Step 1: Solve parameters $\hat{\mathrm{B}}_{x}, \hat{\mathrm{H}}_{y}, \hat{\mathrm{B}}_{z}, \hat{\mathrm{V}}_{x,} \hat{\mathrm{V}}_{y}, \hat{\mathrm{V}}_{z}, \hat{\mathrm{A}}_{x} / \mathrm{f}, \hat{\mathrm{A}}_{y} / \mathbf{f}, \hat{\mathrm{A}}_{z} / \mathbf{f}$, $C_{1} C_{\nabla}$, and $C_{a} / f$ by the proposed algorithm in section 2.2.

Step 2: Compute coordinates $\left(x_{c n}, Y_{c a}, z_{c n}\right)$ obtained by transforming calibration point $\left(x_{n}, y_{n}, z_{n}\right)$ to the camera coordinate system $(\hat{\mathrm{B}}, \hat{\mathrm{v}}, \hat{\mathrm{A}})$ with center $\mathrm{C}$ in Figure 1:

$$
\begin{aligned}
& x_{c D}=\left(x_{x} \hat{A}_{x} / f+Y_{x} \hat{A}_{y} / f+z_{n} \hat{A}_{z} / f-C_{a} / f\right) i_{n} \\
& Y_{c a}=\left(x_{x} \hat{A}_{x} / f+y_{x} \hat{A}_{y} / f+z_{a} \hat{A}_{z} / f-C_{a} / f\right) j_{n} \\
& z_{c n}=\left(X_{x} \hat{A}_{\alpha}+Y_{n} \hat{A}_{y}+z_{n} \hat{A}_{z}-C_{a} \cdot\right)
\end{aligned}
$$

where subscript $\mathrm{n}$ stands for the $\mathrm{n}^{\text {th }}$ calibration point $(n=1, \ldots, N)$.

Step 3: Obtain a $3 \times 3$ matrix $G$ from matrix $G$ for the! $n^{\text {th }}$ calibration point as follows:

$G_{n}=\left[\begin{array}{llll}\left(x_{c n}-\bar{x}_{c}\right)\left(x_{n}-\bar{x}\right) & \left(y_{c n}-\bar{y}_{c}\right)\left(x_{n}-\bar{x}\right) & \left(z_{c n}-\bar{z}_{c}\right)\left(x_{n}-\bar{x}\right) \\ \left(x_{c n}-\bar{x}_{c}\right)\left(y_{n}-\bar{y}\right) & \left(y_{c n}-\bar{y}_{c}\right)\left(y_{n}-\bar{y}\right) & \left(z_{c n}-\bar{z}_{c}\right)\left(y_{n}-\bar{y}\right) \\ \left(x_{c n}-\bar{x}_{c}\right)\left(z_{n}-\bar{z}\right) & \left(y_{c D}-\bar{y}_{c}\right)\left(z_{n}-\bar{z}\right) & \left(z_{c n}-\bar{z}_{c}\right)\left(z_{n}-\bar{z}\right)\end{array}\right]$

where $\left(\bar{x}_{c}, \overline{\mathrm{Y}}_{c}, \bar{z}_{c}\right)$ and $(\overline{\mathrm{x}}, \overline{\overline{\mathrm{Y}}}, \overline{\bar{z}})$ are the respective averages.

$G=\sum_{n=1}^{N} G_{n}$

Step 4: Compute the singular value decomposition of $G$ as $G=U D V$ where $U$ and $V$ are orthonormal and $D$ is a diagonal matrix. 
Step 5: $\quad$ Compute orthonormal $R=V^{2} U^{T}$.

Step 6: From the new extrinsic parameters (obtained from $R$ ) compute focal length $f$ by Step 2 of Method I in Section 2.2.

Step 7: Repeat steps 2-6 for a maximum number of iterations.

\section{Appendix II}

Proof of Derivation of Camera Parameter $f$ in Bquation (9)

The orthonormal rotation matrix $\mathrm{R}$ obtained from the camera matrix in equation (5) is as follows:

$$
R=\left|\begin{array}{lll}
\hat{H}_{x} & \hat{H}_{y} & \hat{H}_{z} \\
\hat{V}_{x x} & \hat{V}_{y} & \hat{V}_{z} \\
A_{y} & \hat{A}_{y} & A_{y}
\end{array}\right|
$$

By the normal properties of the first two columns of the rotation matrix $R$, we obtain the following equation:

$$
\hat{\mathrm{B}}_{x} \hat{\mathrm{B}}_{y}+\hat{\mathrm{V}}_{x} \hat{\mathrm{V}}_{y}+\hat{\mathrm{A}}_{x} \hat{\mathrm{A}}_{y}=0
$$

The parameters computed from the matrix equation (4) are $\hat{\mathrm{H}}_{\mathrm{x}}$, $\hat{\mathrm{H}}_{y}, \hat{\mathrm{V}}_{\mathbf{x}}, \hat{\mathrm{V}}_{\mathrm{y}}$ and $\boldsymbol{C}_{\mathrm{v}}$. The parameters computed from equation (8) are $\hat{A}_{x} / f, \hat{A}_{y} / f$ and $C_{a} / f$. From these known parameters, we can rearrange equation (30) above to obtain equation (9).

Proof of Derivation of Camera Parameter $C_{h}$ in Equation (12)

Since the first two columns of the rotation matrix $R$ in equation (29) above are unit vectors, we can obtain the following equations :

$$
\hat{\mathrm{H}}_{x}^{2}+\hat{\mathrm{V}}_{x}^{2}+\mathrm{f}^{2}\left(\hat{\mathrm{A}}_{\mathrm{x}} / f\right)^{2}=1
$$

or 


$$
\hat{\mathrm{B}}_{\mathrm{y}}^{2}+\hat{\mathrm{V}}_{\mathrm{y}}^{2}+\mathrm{f}^{2}\left(\hat{\mathrm{A}}_{\mathrm{y}} / f\right)^{2}=1
$$

Since parameters $\hat{\mathrm{B}}_{\mathrm{x}}, \hat{\mathrm{B}}_{\mathrm{y}}, \hat{\mathrm{V}}_{\mathrm{x}}, \hat{\mathrm{V}}_{\mathrm{y}}, \hat{\mathrm{A}}_{\mathrm{x}} / \mathbf{f}$ and $\hat{\mathrm{A}}_{\mathrm{y}} / \mathbf{f}$ obtained from equations (4) and (8) assume that $C_{b}=1$, we can obtain the value of $C_{h}$ from equation (31) as shown in equation (10).

\section{Appendix III}

\section{Proofs of Theorems 1 and 2}

Let $M$ be the camera matrix, with no image center displacement and scale factor, in equation (5) for the 3D calibration case.

Let $\mathbf{M}_{i j}(1 \leq i, j \leq 3)$ be the entries of the matrix M. Including image center displacement $\left(i_{0}, j_{0}\right)$ and scale factor $\left(\boldsymbol{s}_{1}, \boldsymbol{s}_{j}\right)$ the new camera matrix $\mathbf{M}^{\prime}$ with entries $\mathbf{M}_{1 j}^{\prime}(1 \leq i, j \leq 3)$ can be expressed in terms of $\mathbf{M}_{1,}$ from equations (12) and (14) as follows:

$$
M^{\prime}=\left[\begin{array}{cccc}
s_{1}, M_{11}+i_{0} M_{31} & s_{1} M_{12}+i_{0} M_{32} & s_{1} M_{13}+i_{0} M_{33} & s_{1} M_{14}+i_{0} M_{34} \\
s_{f} M_{21}+j_{0} M_{31} & s_{f} M_{22}+j_{0} M_{32} & s_{f} M_{23}+j_{0} M_{33} & s_{f} M_{24}+j_{0} M_{34} \\
M_{31} & M_{32} & M_{33} & M_{34}
\end{array}\right]
$$

Let $\left(\boldsymbol{i}_{\boldsymbol{f}}, \boldsymbol{j}_{\boldsymbol{f}}\right)$ be the image coordinates (not corrected for image center displacement and scale factor) of a world point $(\mathbf{x}, \mathbf{y}, \mathbf{z})$. Let $\left(i^{\prime}, j^{\prime}\right)$ be the computed image coordinates from world coordinates $(x, y, z)$ with camera matrix M'. From equations (6) and (11), (j.', $\left.j^{\prime}\right)$ can be expressed as follows: 


$$
\begin{aligned}
i^{\prime} & =\frac{\left(s_{1} M_{11}+i_{0} M_{31}\right) x+\left(s_{1} M_{12}+i_{0} M_{32}\right) y+\left(s_{1} M_{13}+i_{0} M_{33}\right) z+\left(s_{1} M_{14}+i_{0} M_{34}\right)}{\left(M_{31} x+M_{32} y+M_{33} z+M_{34}\right)} \\
& =s_{1} i^{i}+i_{0}=i_{t} \\
j^{\prime} & =\frac{\left(s_{j} M_{21}+j_{0} M_{31}\right) x+\left(s_{j} M_{22}+j_{0} M_{32}\right) y+\left(s_{j} M_{23}+j_{0} M_{33}\right) z+\left(s_{j} M_{24}+j_{0} M_{34}\right)}{\left(M_{31} x+M_{32} y+M_{33} z+M_{34}\right)} \\
& =s_{j} j+j_{0}=j_{t}
\end{aligned}
$$

where $(i, j)$ are the image coordinates corrected for image center displacement and scale factor of world point $(x, y, z)$.

The above expressions show that the computed image coordinates $\left(i^{\prime}, j^{\prime}\right)$ are same as the uncompensated image! coordinates $\left(i_{f}, j_{f}\right)$ of world point $(x, y, z)$. This proves; Theorem 1. This result can be extended to the $2 \mathrm{D}$ calibration case by starting from the camera matrix $M$ in equation (6).

For the 2D calibration case, let $N=M^{-1}$ where $M$ is the camera matrix in equation (6). Let $N_{i j}(1 \leq i, j \leq 3)$ be the! entries of matrix $N$. Let $N^{\prime}=\left(M^{\prime}\right)^{-1}$ where $N^{\prime}{ }_{i j}(1 \leq i, j \leq 3)$ are entries of matrix N. From equation (32) we obtain the following expressions :

$$
\begin{aligned}
& \mathbf{N}^{\prime}{ }_{11}=\mathbf{s}_{1}^{-1} \mathbf{N}_{11} \\
& N^{\prime}{ }_{21}={B_{1}}^{-1} \mathbf{N}_{21} \\
& N^{\prime}{ }_{31}=s_{1}^{-1} N_{31} \\
& \mathrm{~N}^{\prime}{ }_{12}=\mathrm{s}_{\mathrm{j}}{ }^{-1} \mathrm{~N}_{12} \\
& \mathbf{N}^{\prime}{ }_{22}=s_{j}{ }^{-1} \mathbf{N}_{22} \\
& \mathbf{N}^{\prime}{ }_{32}=s_{j}^{-1} \mathbf{N}_{32} \\
& \mathrm{~N}_{13}^{\prime}=\mathrm{N}_{13}-\mathrm{s}_{1}^{-1} \mathrm{i}_{0} \mathrm{~N}_{11}-\mathrm{B}_{j}{ }^{-1} \mathrm{j}_{0} \mathrm{~N}_{12} \\
& N^{\prime}{ }_{23}=N_{23}-s_{1}{ }^{-1} i_{0} N_{21}-s_{j}^{-1} j_{0} N_{22} \\
& N^{\prime}{ }_{33}=N_{33}-B_{1}^{-1} i_{0} N_{31}-8_{j}^{-1} j_{0} N_{32}
\end{aligned}
$$


Iet; $\left(i_{f}, j_{f}\right)$ be the image coordinates (not compensated for image center displacement and scale factor) of an world point $(x, y)$. Let $\left(x^{\prime}, y^{\prime}\right)$ be the computed world coordinates from image coordinates $\left(i_{f}, j_{f}\right)$ with camera matrix $N^{\prime}$. From equations (6), (33) and (11) we obtain the following expressions:

$$
\begin{aligned}
x^{\prime} & =\frac{s_{1}^{-1} N_{11} i_{f}+s_{j}^{-1} N_{12} j_{f}+\left(N_{13}-s_{1}^{-1} N_{11} i_{0}-s_{j}^{-1} N_{12} j_{0}\right)}{s_{1}^{-1} N_{31} i_{f}+s_{j}^{-1} N_{32} j_{f}+\left(N_{33}-s_{1}^{-1} N_{31} i_{0}-s_{j}^{-1} N_{32} j_{0}\right)} \\
& =\frac{s_{1}^{-1}\left(i_{f}-i_{0}\right) N_{11}+s_{j}^{-1}\left(j_{f}-j_{0}\right) N_{12}+N_{13}}{s_{1}^{-1}\left(i_{f}-i_{0}\right) N_{31}+s_{j}^{-1}\left(j_{f}-j_{0}\right) N_{32}+N_{33}} \\
& =\frac{N_{11} i+N_{12} j+N_{13}}{N_{31} i+N_{32} j+N_{33}}=x \\
y^{\prime} & =\frac{s_{1}^{-1} N_{21} i_{f}+s_{j}^{-1} N_{22} j_{f}+\left(N_{23}-s_{1}^{-1} N_{21} i_{0}-s_{j}^{-1} N_{22} j_{0}\right)}{s_{1}^{-1} N_{31} i_{f}+s_{j}^{-1} N_{32} j_{f}+\left(N_{33}-s_{1}^{-1} N_{31} i_{0}-s_{j}^{-1} N_{32} j_{0}\right)} \\
& =\frac{s_{1}^{-1}\left(i_{f}-i_{0}\right) N_{21}+s_{j}^{-1}\left(j_{f}-j_{0}\right) N_{22}+N_{23}}{s_{1}^{-1}\left(i_{f}-i_{0}\right) N_{31}+s_{j}^{-1}\left(j_{f}-j_{0}\right) N_{32}+N_{33}} \\
& =\frac{N_{21} i+N_{22} j+N_{23}}{N_{31} i+N_{32} j+N_{33}}=y
\end{aligned}
$$

The above equations show that the computed world coordinates $\left(x^{\prime}, y^{\prime}\right)$ are same as the true world coordinates $(x, y)$ for the $2 D$ calibration case. This proves Theorem 2. This result for the 2D case can be extended to include Method II of the 3D calibration case for a stereo setup. 


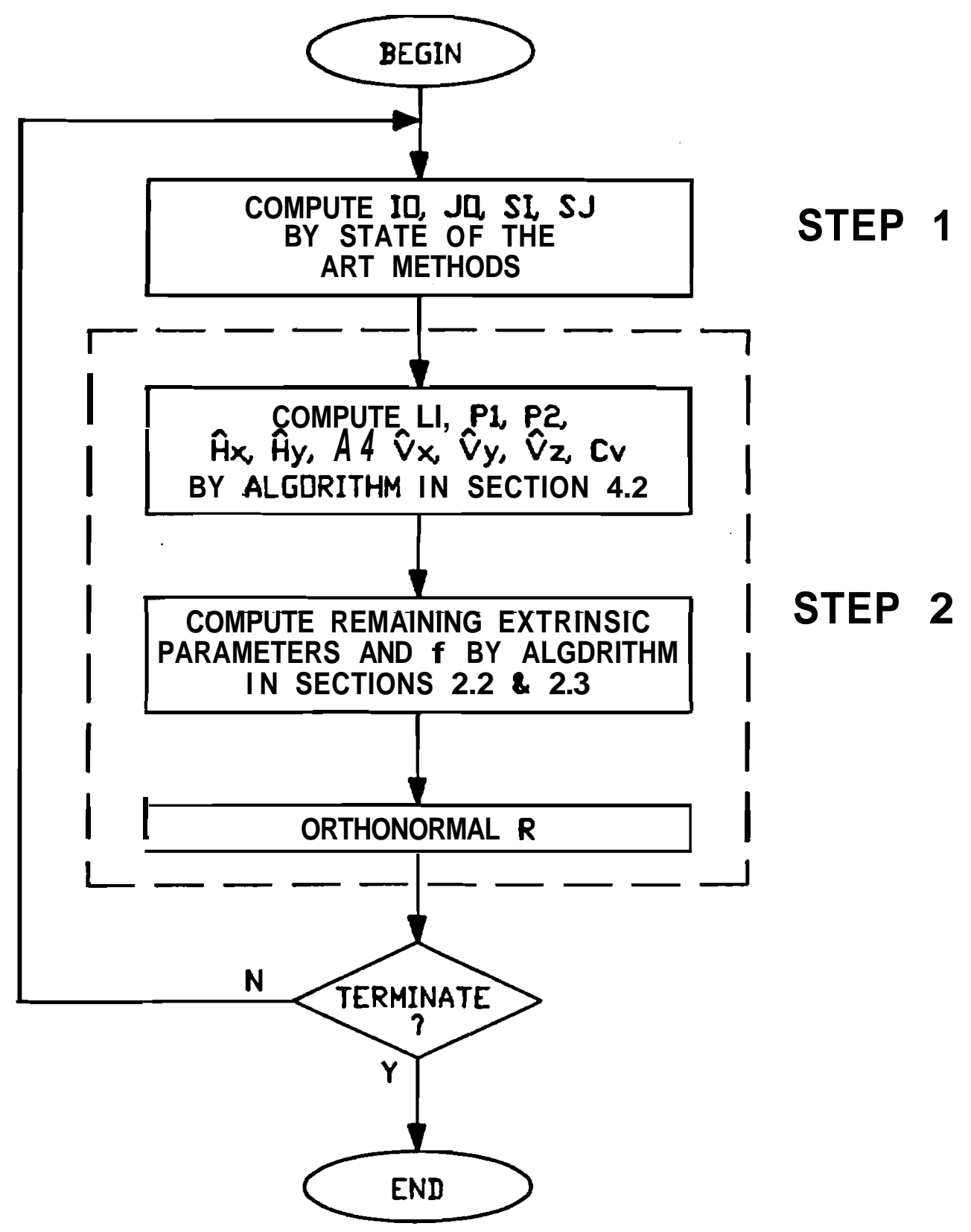

FIGURE 3. FLDWCHART FDR THE COMPLETE ALGIRITHM FUR THE COMPUTATION OF ALL CALIBRATIDN PARAMETERS. 

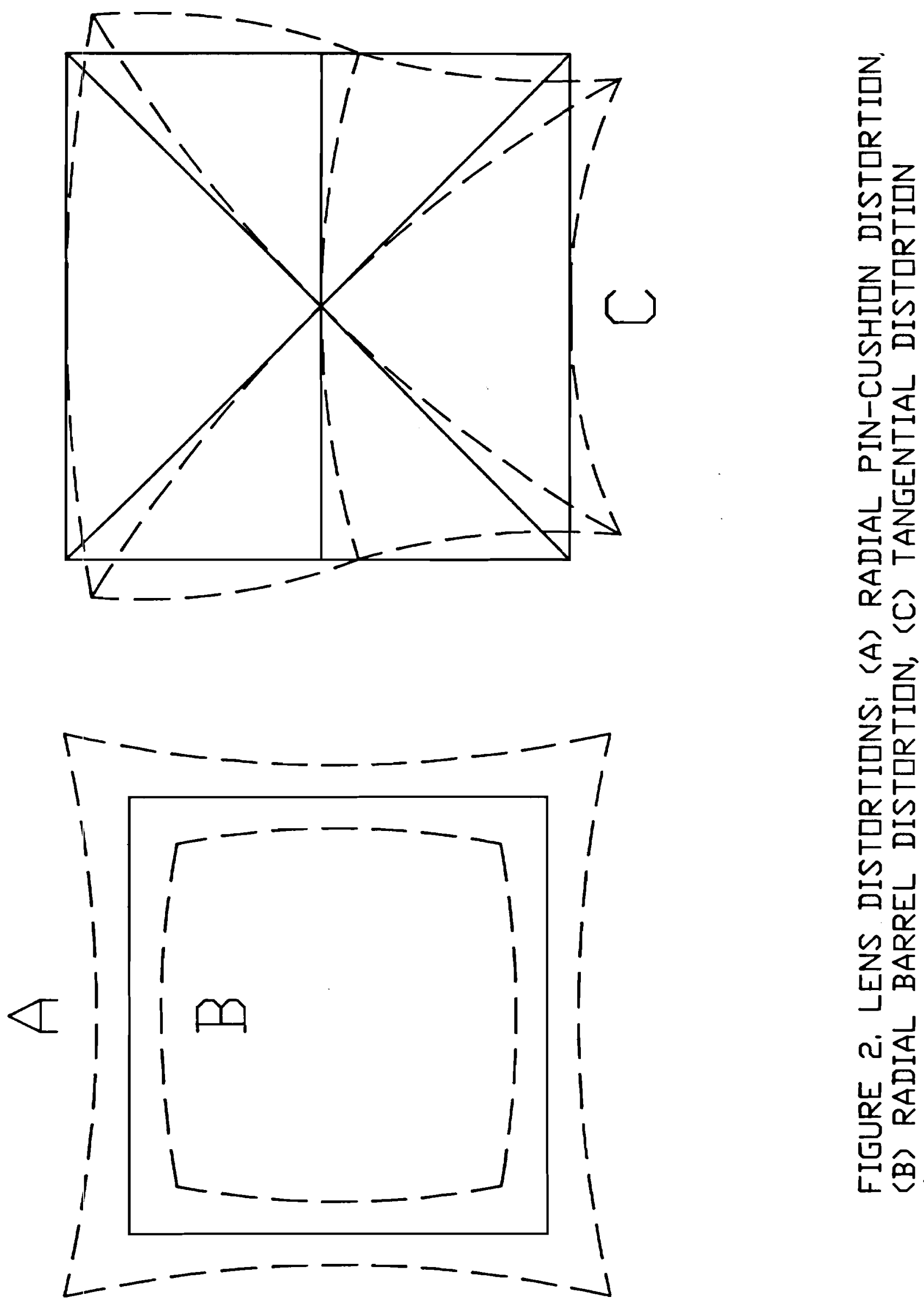

记䔎 


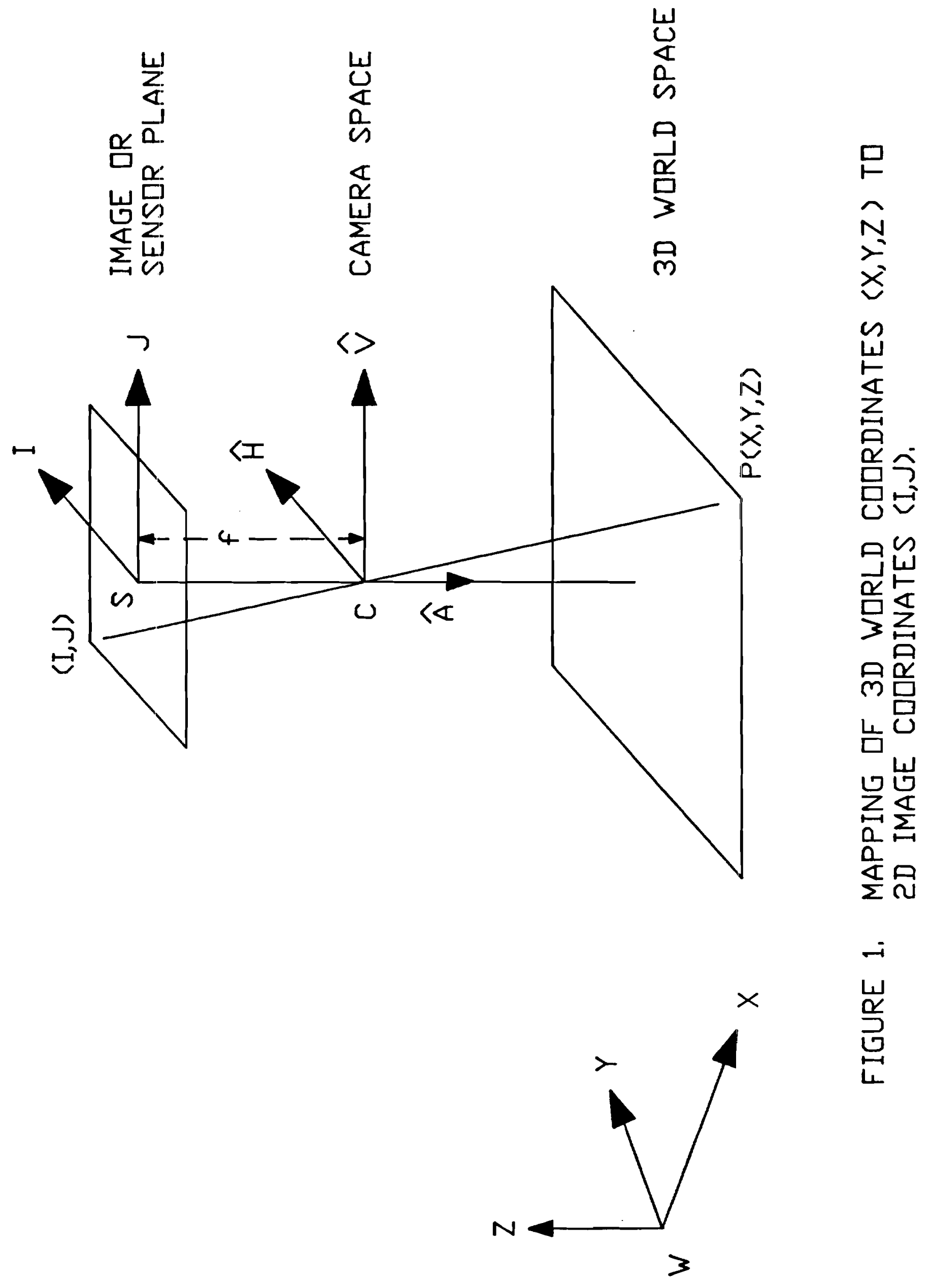




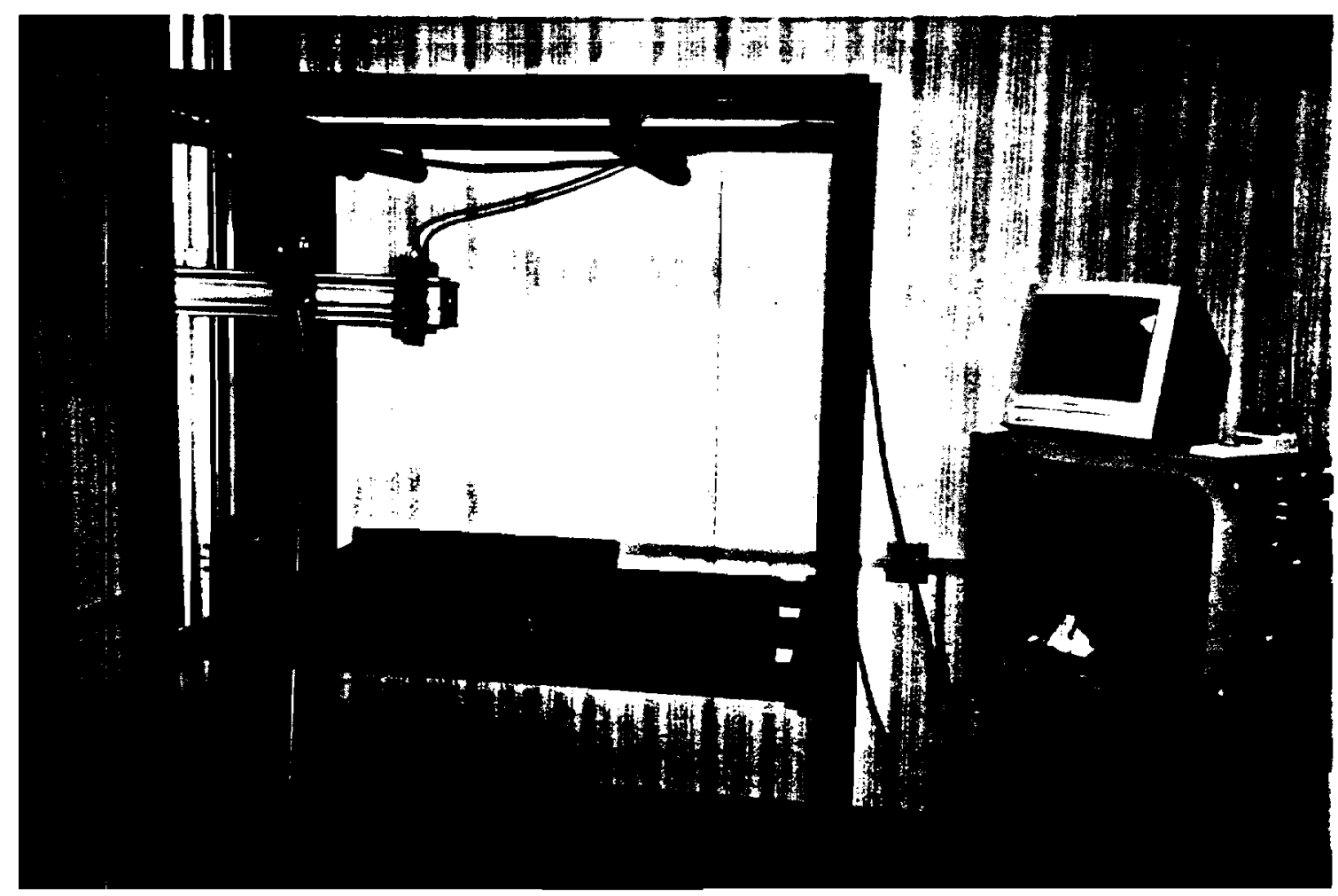

FIGURE 4. SETUP FOR CAKRRA CALIBRATION 